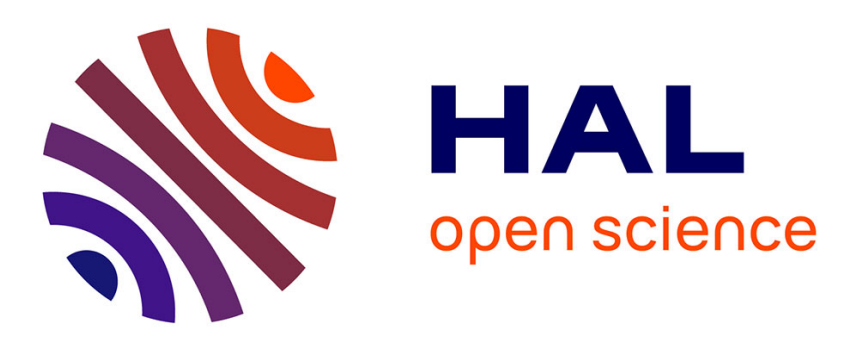

\title{
3-d topology optimization of modulated and oriented periodic microstructures by the homogenization method
}

Perle Geoffroy-Donders, Grégoire Allaire, Olivier Pantz

\section{To cite this version:}

Perle Geoffroy-Donders, Grégoire Allaire, Olivier Pantz. 3-d topology optimization of modulated and oriented periodic microstructures by the homogenization method. Journal of Computational Physics, 2020, 401, pp.108994. hal-01939201

\section{HAL Id: hal-01939201 https://hal.science/hal-01939201}

Submitted on 29 Nov 2018

HAL is a multi-disciplinary open access archive for the deposit and dissemination of scientific research documents, whether they are published or not. The documents may come from teaching and research institutions in France or abroad, or from public or private research centers.
L'archive ouverte pluridisciplinaire HAL, est destinée au dépôt et à la diffusion de documents scientifiques de niveau recherche, publiés ou non, émanant des établissements d'enseignement et de recherche français ou étrangers, des laboratoires publics ou privés. 


\title{
3-d topology optimization of modulated and oriented periodic microstructures by the homogenization method
}

\author{
Perle Geoffroy-Donders* $\quad$ Grégoire Allaire ${ }^{\dagger} \quad$ Olivier Pantz ${ }^{\ddagger}$
}

November 13, 2018

\begin{abstract}
This paper is motivated by the optimization of so-called lattice materials which are becoming increasingly popular in the context of additive manufacturing. Generalizing our previous work in 2 -d we propose a method for topology optimization of structures made of periodically perforated material, where the microscopic periodic cell can be macroscopically modulated and oriented. This method is made of three steps. The first step amounts to compute the homogenized properties of an adequately chosen parametrized microstructure (here, a cubic lattice with varying bar thicknesses). The second step optimizes the homogenized formulation of the problem, which is a classical problem of parametric optimization. The third, and most delicate, step projects the optimal oriented microstructure at a desired length scale. Compared to the 2 -d case where rotations are parametrized by a single angle, to which a conformality constraint can be applied, the 3 -d case is more involved and requires new ingredients. In particular, the full rotation matrix is regularized (instead of just one angle in 2-d) and the projection map which deforms the square periodic lattice is computed component by component. Several numerical examples are presented for compliance minimization in 3-d.
\end{abstract}

\section{Introduction}

The homogenization method is the ancestor of many popular and successful algorithms for topology optimization of structures. Its main idea is to introduce microstructures with a continuously variable material density as admissible designs in the optimization process. This idea was first introduced by mathematicians [19], [23], [25] (see the textbook [1] for more references), who motivated the use of such composite materials by the notion of relaxation, i.e., making the

\footnotetext{
*Safran Tech, Magny-les-Hameaux, France - perle.geoffroy@polytechnique.edu

${ }^{\dagger}$ CMAP, École Polytechnique, Palaiseau, FRANCE - gregoire.allaire@polytechnique.fr

‡Université Côte d'Azur, LJAD, FRANCE - pantz@unice.fr
} 
optimization well-posed. The homogenization method became popular thanks to the pioneering paper [9] which was the first one to numerically address a realistic problem in the elasticity setting. Since then, the homogenization method has been replaced by its much simplified version, the so-called SIMP method introduced in [8], [35] (see the textbook [10] for a more complete account), which is the most commonly used method in commercial topology optimization software, as well as in many academic contributions. Compared to the homogenization method, which relies on true composite materials, possibly anisotropic, SIMP uses only fictitious isotropic materials. Since intermediate densities (between full material and void) are penalized in the end, there is indeed no need to have a detailed knowledge and optimization of microstructures.

Nevertheless, the recent progress of additive manufacturing techniques revive the interest for the use of graded or microstructured materials since they are now manufacturable. Their range of applications is very large, from standard lightweighting mechanical structures [32], to the design of bone scaffolds [12, 18], passing by heat exchangers [22].

Although homogenization theory applies to any kind of composite materials (without any restriction on the geometry of their microstructure), in this work we restrict ourselves to periodic homogenization and macroscopically modulated periodic structures. The reason for this choice is the obvious manufacturability of such periodic structures, although other choices would be possible, like (stationary) random structures (see e.g. [24]). The optimization of periodic microstructure for composite materials is an old topic, still very active, see e.g. [5], [6], [7], [16], [21], [30], [31], [33], [34]. Typically, the properties of the microstructures are homogenized (or averaged), then parametrized in order to optimize only a few scalar fields. Very often, these microstructures are anisotropic. However, their orientation is rarely taken into account and optimized, although it is well-known that their orientation is a crucial and determining parameter in topology optimization [1,28]. Actually, if optimizing the microstructure orientation is not difficult, reconstructing the oriented periodic structure is a challenging issue. In particular, two neighbouring cells, if oriented differently, either overlap or leave a small gap between their sides. Therefore, the periodic structure might be not connected or would not respect an imposed volume constraint [36, 11]. Morphing approaches have been suggested [31], but they are not effective as soon as the cell orientation varies in the structure. Another method, developed in 2-d [27, 2, 15] alleviates this difficulty by deforming the original periodic grid with a diffeormorphism, which ensures the cohesion between cells by slightly distorting them. This diffeormorphism is defined and computed in such a way that, roughly speaking, its gradient is aligned with the optimal orientation of the periodicity cell.

The goal of the present paper is to extend this approach from the 2-d setting to the 3-d case. It is not a simple matter since many new difficulties appear, which are not merely computational issues but also modeling and theoretical issues. In particular, orientation in 2 -d is easily parametrized by a single angle which, furthermore, can satisfy a conformality condition, ensuring preservation of angles upon deformation. Such a conformality condition is a too restrictive 
requirement in 3-d and there are several different ways of representing orientation. Here, orientation would be represented by a rotation matrix. There are two main novelties in the present work. First, we propose a new method to regularize the orientation of the cells in 3-d, which avoids the sign indeterminacy of a vector representing a direction (see Section 4.3). Second, we extend in 3-d our 2-d projection method [2] in order to reconstruct a modulated and oriented periodic structure with properties closed to the homogenized optimal design. The key difference with respect to the 2-d setting is that the projection is made direction by direction and not globally (see Section 5 ).

The content of this paper is the following. Section 2 introduces the optimal design problem of compliance minimization in $3-\mathrm{d}$ and its relaxation using the homogenization approach.

Section 3 is devoted to the first or pre-processing step of our method. It amounts to choose a parametrized periodicity cell, here a cube drilled from end to end by three rectangular holes, and to compute its homogenized elasticity properties, as well as their derivatives, with respect to the cell parameters. We also discuss a representation of the cell orientation by a set of three orthogonal unit vectors (in 3-d) in Section 3.3.

Section 4 focuses on the second step of our method, namely the optimization of the homogenized formulation of the 3 -d compliance problem, with respect to the periodicity cell parameters. We rely on a gradient-based algorithm where the gradient is classically computed by an adjoint approach. The optimal orientation is found analytically [28], [26] since we consider single load compliance minimization problem. However, for more general problems we could have used a more standard, albeit less efficient, gradient algorithm for the orientation (see Remark 2). The method differs from the one developed in 2-d [2] by the regularization of the cell orientation. Indeed, after optimization, the orientation has to be slightly regularized in order to lead to reasonable results during the post-processing step. The regularization method, presented in Section 4.3 is one of the main novelties of the present work. It relies on the fact that the chosen orientation representation is not unique: each three vectors can be replaced by its opposite. Hence, the regularization approach has to take into account this property. A symmetric matrix, with eigenvectors given by these three orientation vectors, is introduced. The regularization of this matrix is then preferably chosen, rather than a direct regularization of the orientation vectors.

Section 5 deals with the third or post-processing step, namely the projection of the homogenized design over genuine shapes. As in 2-d [2], a vector field $\varphi(x)$, which distorts a regular grid according to a given local orientation, is defined. The main difference with 2-d is that the map $\varphi$ is no longer a conformal map, which would be a too restrictive requirement in 3-d. Section 5.2 introduces a method to compute the map $\varphi$. Numerical implementation is detailed in Section 5.4. The numerical results are displayed in Section 5.5 for a cantilever test case. We emphasize that the orientation regularization and the post processing algorithm are completely general, meaning that they are not restricted to single load compliance minimization, and that they can readily be implemented for any other optimization problem [14]. 
In Section 6 numerical examples of the whole process applied to a bridge and to an electrical mast are displayed.

\section{Setting of the problem}

Let $D \subset \mathbb{R}^{3}$ be a fixed smooth bounded open set (the working domain) and $\Omega \subset D$ the reference configuration of an isotropic elastic body. The structure $\Omega$ is clamped on $\Gamma_{D} \subset \partial \Omega$, and submitted to surface loads $g$ on $\Gamma_{N} \subset \partial \Omega$. For simplicity these parts $\Gamma_{D}$ and $\Gamma_{N}$ of the boundary are assumed to be given subsets of $\partial D$, independent of $\Omega$. We assume that the solid is made of an homogeneous isotropic linear elastic material of Hooke's law $A$, with Lamé coefficients $\lambda$ and $\mu$. The displacement $u$ and the stress tensor $\sigma$ are then solutions of the system

$$
\begin{cases}\operatorname{div}(\sigma)=0 & \text { in } \Omega \\ \sigma=A e(u) & \text { in } \Omega, \\ u=0 & \text { on } \Gamma_{D} \\ \sigma \cdot n=g & \text { on } \Gamma_{N} \\ \sigma \cdot n=0 & \text { on } \Gamma=\partial \Omega \backslash\left(\Gamma_{D} \cup \Gamma_{N}\right),\end{cases}
$$

where $e(u)=\frac{1}{2}\left(\nabla u+\nabla u^{T}\right)$ is the strain tensor (the symmetrized gradient of the displacement). Shape and topology optimization consists in determining the domain $\Omega$ that minimizes a given objective function $J$,

$$
\begin{aligned}
& \min _{|\Omega| \leq V,} J(\Omega) \\
& \Gamma_{D} \cup \Gamma_{N} \subset \partial \Omega
\end{aligned}
$$

where $V \in \mathbb{R}^{+}$is the maximum admissible volume. A typical objective function is the compliance

$$
J(\Omega)=\int_{\Gamma_{N}} g \cdot u d s .
$$

As is well known [1], for most cost functions $J$, problem (1) does not admit a solution. This is due to the fact that composite shapes, made of very small microstructures, can always outperform genuine shapes made of plain material. A composite shape is described by the local density $\theta(x)$ of material and a homogenized elasticity tensor $A^{*}(x)$ that depends on the microstructure at the point $x \in D$. The homogenized or macroscopic displacement $u^{*}$ of the structure is then solution of the system

$$
\begin{cases}\operatorname{div}(\sigma)=0 & \text { in } D \\ \sigma=A^{*} e\left(u^{*}\right) & \text { in } D \\ u^{*}=0 & \text { on } \Gamma_{D} \\ \sigma \cdot n=g & \text { on } \Gamma_{N} \\ \sigma \cdot n=0 & \text { on } \Gamma=\partial D \backslash\left(\Gamma_{D} \cup \Gamma_{N}\right) .\end{cases}
$$

We emphasize that the mechanical problem is now defined on the whole working domain $D$ and no longer on a shape $\Omega \subset D$. Then, the minimization problem 
should be rewritten as a minimization problem of a relaxed cost function $J^{*}$ with respect to the homogenized elasticity tensor $A^{*}$ and the density $\theta$

$$
\begin{gathered}
\min _{\substack{\int_{D} \theta(x) d x \leq V, A^{*}(x) \in G_{\theta}(x) \text { a.e. } x \in D}} J^{*}\left(\theta, A^{*}\right),
\end{gathered}
$$

where $G_{\theta}(x)$ is the set of effective or homogenized Hooke's laws for microstructures of density $\theta(x)$. The main difficulties in solving the homogenized formulation (2) are, first, to compute the relaxed cost function $J^{*}$ (which may be different from the original cost function $J$ ), second and more importantly, to give a complete and explicit description of the set of admissible Hooke's laws $G_{\theta}$. It is only for special cases (like compliance minimization) that (2) can be made fully explicit [1]. Furthermore, composite shapes are only ideal mathematical objects. Very often, optimal composites are multi-scale microstructures (like sequential laminates) which cannot be actually built as they are made of infinitely small details. To circumvent these obstacles, we restrict the set of admissible composites to periodic microstructures for which the Hooke's law can be numerically computed and, furthermore, which can be explicitly projected on a so-called lattice structure as in [2], [27]. Of course, optimizing periodic composites is an old idea that goes back at least to [9]. The new idea here is that this periodic microstructure will be projected to build a sequence of genuine non composite shapes with increasing levels of detail that converges toward the optimal periodic composite.

\section{Preprocessing : homogenized Hooke's laws of the microstructures}

\subsection{Set of admissible microstructures}

From now on, we limit our analysis to a simple class of periodic composites, which is a natural extension to 3 -d of the one used in the seminal paper [9]. The periodic cell is the unit cube drilled from face to face by three cylindrical holes with rectangular cross sections (see Figure 1). Hence this class of composites is parametrized by its geometric parameters $m=\left(m_{1}, m_{2}, m_{3}\right)$, where $\left(m_{i-1}, m_{i+1}\right)$ denotes the size of the holes rectangular cross sections perpendicular to direction $e_{i}$. We emphasize the fact that we only have three geometric parameters, since each hole has a common dimension with each of the two remaining holes. The periodic cell with geometric parameters $m$ is denoted $Y(m)$. The solid volume fraction in $Y(m)$ is $\theta(m)=1-m_{1} m_{2}-m_{1} m_{3}-m_{2} m_{3}+2 m_{1} m_{2} m_{3}$. A mathematically more precise definition of the cell $Y(m)$ is given in Section 5.1. Moreover, the cubic cell can be freely oriented in the space. This orientation is denoted by $\omega$ (an explicit definition of $\omega$ will be given later in Section $3.3)$.

Rank 3-laminates are known to reach the optimum for single-load compliance minimization problems [1]. The directions of lamination are given by the prin- 
cipal direction of the stress tensor and, consequently, form an orthogonal basis. There are two regimes for the optimal proportions of lamination. First, where the stress tensor is quite isotropic, the optimum laminate is a non degenerate rank-3 laminate. This microstructure features closed holes. Second, where the stress tensor is strongly anisotropic (the principal stress in one direction is much larger than in the orthogonal plane), the optimum is reached by a degenerate rank-3 laminate, namely a rank-2 laminate. Such a microstructure looks like tubular holes in the direction of the largest principal stress. In any case, those laminates feature up to three well separated microscopic scales and thus they are far from being manufacturable, even by additive manufacturing processes. Hence we have to choose a simpler microstructure, featuring a single microscopic scale, probably sub-optimal but manufacturable. Another reason for choosing the periodic cell of Figure 1 is that it does not contain closed holes (contrary to rank-3 laminates) and thus the metal powder can be recovered after the building process. Indeed, another possible periodic cell, which would be more similar to a rank-3 laminate, is a cubic cell with solid walls and a closed hole. However it is not manufacturable since the metal powder would be trapped in the closed hole and could not be removed Hence, our choice of periodic cell is not the union between three orthogonal planes, but their intersection, resulting in through holes. Obviously, this microstructure is not optimal, and could be improved (for example, by rounding its corners and edges to avoid local stress concentrations), possibly at the detriment of the manufacturability. Possibly, other manufacturable microstructures could be considered. For example a generalization of a rank-2 laminate (from the second regime), namely a material featuring through tubular holes, may be relevant, although not optimal, in the single-load compliance minimization problem. Other manufacturable orthotropic materials, dedicated to additive manufacturing are developed [24] and could be good candidates too. We emphasize that orthotropic periodic materials whose elastic properties can be modulated through cell parameters in three orthogonal directions separately, like rank-3 laminates, are the best candidates for singleload compliance minimization problems. Indeed, in our numerical experiments, see [2], compliance minimization with isotropic cells, like Kelvin foams [20], or orthotropic cells modulated only by density, leads to black and white designs (with almost no composite zones of intermediate densities), less optimal than the structures computed by the present method. In other words, these microstructures are self-penalizing (a feature already remarked for isotropic materials in [4]). In any case, the present optimization method could be implemented for other orthotropic composites without any additional work.

Remark 1. Recall that we focus here on single-load compliance minimization problems (see Remark 2). For other objective functions, suitable microstructures are not discussed here and should be investigated. 


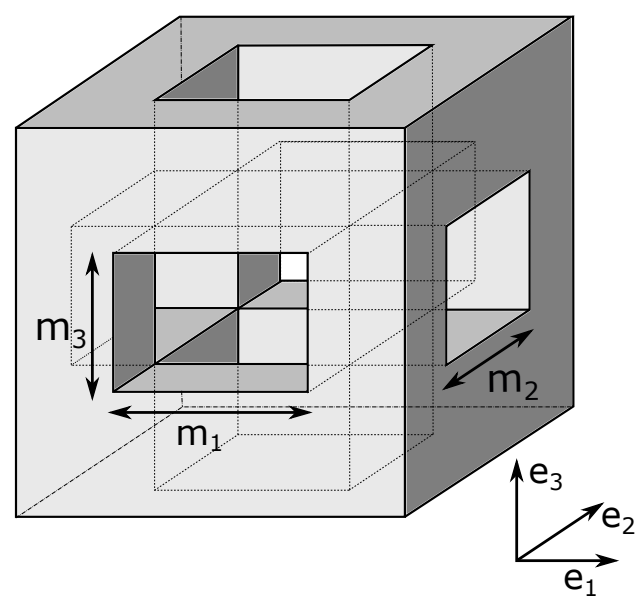

Figure 1: Periodicity cell $Y(m)$.

\subsection{Cell problem and homogenized elasticity tensor}

The periodic cell $Y(m)$ is the unit cube perforated by holes, the boundary of which is denoted by $\Gamma_{\text {int }}$. It defines a composite material which has a macroscopic of effective behavior characterized by an homogenized elasticity tensor $A^{*}$. In other words, the local geometry of the cell $Y(m)$ is enough to describe the elastic properties of the composite material. More detailed explanations about the homogenization method could be found in [1].

To compute this homogenized tensor $A^{*}$, one needs so-called correctors $w_{i j}$, corresponding to the local displacements in the cell $Y(m)$, defined for each pair $(i, j) \in\{1,2,3\}^{2}$ as the solutions of the following cell problem

$$
\left\{\begin{array}{cl}
\operatorname{div}\left(A\left(e_{i j}+e\left(w_{i j}\right)\right)\right)=0 & \text { in } Y(m) \\
A\left(e_{i j}+e\left(w_{i j}\right)\right) n=0 & \text { on } \Gamma_{i n t} \\
y \mapsto w_{i j}(y) & (0,1)^{3} \text {-periodic }
\end{array}\right.
$$

where $e_{i j}=\frac{1}{2}\left(e_{i} \otimes e_{j}+e_{j} \otimes e_{i}\right)$ is a basis of the symmetric tensors of order 2 and $n$ is the normal to the hole boundaries $\Gamma_{i n t}$. This problem admits a unique solution, up to an additive translation. The variational formulation of the cell problem (3) consists in finding $w_{i j} \in H_{\#}^{1}\left(Y(m), \mathbb{R}^{3}\right)$ such that

$$
\forall \phi \in H_{\#}^{1}\left(Y(m), \mathbb{R}^{3}\right) \quad \int_{Y(m)} A e\left(w_{i j}\right): e(\phi)+\int_{Y(m)} A e_{i j}: e(\phi)=0 .
$$

The tensor $A^{*}$ is then given in terms of the solutions $w_{i j}$ of the cell problems by

$$
A_{i j k l}^{*}=\int_{Y(m)} A\left(e_{i j}+e\left(w_{i j}\right)\right):\left(e_{k l}+e\left(w_{k l}\right)\right) d y \quad \forall i, j, k, l \in\{1,2,3\} .
$$


The above equation defines indeed a coercive fourth order tensor with the suitable symmetric properties of an elasticity tensor.

Since the geometry of the cell $Y(m)$ depends on the parameter $m$, the corresponding homogenized elastic tensor depends on the parameters $m$ too, and it is denoted $A^{*}(m)$. The sensitivity of the homogenized tensor $A^{*}(m)$, with respect to $m$, is given by the same formula than in 2-d, (see [2], equation (6)). Since, by virtue of its definition (5), $A_{i j k l}^{*}$ is an energy, computing the sensitivity of $A^{*}(m)$ is a self-adjoint problem (no adjoint are necessary) and the derivative formula is easily obtained by differentiating (5) with respect to the shape.

\subsection{Cell orientation}

The periodic cells $Y(m)$ may be oriented and their orientation can vary in the working domain $D$. Indeed, they are orthotropic: they feature three orthogonal planes of symmetry. Therefore, their elastic behavior depends also on their 3-d orientation, denoted $\omega$. The homogenized elastic tensor will be denoted $A_{\omega}^{*}(m)$ (the orientation is in subscript in the chosen notation). Indeed, thanks to a rotation operator, the homogenized tensor $A_{\omega}^{*}(m)$ can easily be computed for any orientation $\omega$ from $A_{\omega_{0}}^{*}(m)$, where $\omega_{0}$ is an arbitrary orientation. Consequently, if the orientation is fixed, the homogenized tensor can be seen as depending only on the geometric parameters. On the contrary, the geometric parameter $m$ is an intrinsic variable of the elasticity tensor.

The modeling of the cell orientation in 3-d is a delicate problem: one could use Euler angles or quaternions for example. However our choice is dictated by the optimization method. Indeed, the optimal orientation in a compliance minimizing problem with orthotropic cells is given by the three principal directions of the stress tensor (see Section 4.1). Hence, we view the orientation $\omega$ as the set of three unit vectors aligned with the principal directions: $\omega=\left(\omega_{1}, \omega_{2}, \omega_{3}\right)$. Those vectors form an orthonormal basis, since they are eigenvectors of a symmetric matrix, here the stress tensor.

The reference orientation of the cell is the canonical basis $\omega_{0}=\left(e_{1}, e_{2}, e_{3}\right)$, as in Figure 1. For simplicity, we set $A_{\omega_{0}}^{*}(m)=A^{*}(m)$.

Let $S O(3)$ be the special orthogonal group, and let $Q(\omega) \in S O(3)$ be the rotation matrix, whose columns are respectively the unit vectors $\omega_{1}, \omega_{2}$ and $\pm \omega_{3}$. We emphasize that in order to define a rotation matrix (i.e. an element of $S O(3)$ ), we have to ensure that its determinant is equal to 1 , and not to -1 : this defines the sign of $\pm \omega_{3}$. Eventually $Q(\omega)$ is the rotation matrix between the orientations $\omega_{0}$ and $\omega$.

Let $R(\omega)$ be the fourth-order tensor defined by:

$$
\forall \xi \in \mathcal{M}_{3}^{s} \quad R(\omega) \xi=Q(\omega)^{T} \xi Q(\omega),
$$

where $\mathcal{M}_{3}^{s}$ is the set of $3 \times 3$ symmetric matrices. The dependency of $A_{\omega}^{*}(m)$ with respect to the orientation $\omega$ can be made explicit as follows:

$$
A_{\omega}^{*}(m)=R(\omega)^{T} A^{*}(m) R(\omega) .
$$


We emphasize that the knowledge of $R(\omega)$ does not define uniquely $\omega$, since the vectors $\omega_{i}$ are chosen arbitrarily up to their sign. This particularity is inherited from the principal directions of the stress tensor. However, the signs of the vectors $\omega_{i}$ have no impact on the result of equation (6).

\subsection{Numerical implementation}

The set of effective elasticity tensors $\left\{A^{*}(m)\right.$ such that $\left.m \in[0,1]^{3}\right\}$ has to be characterized. The proposed strategy consists in computing the material properties for a discrete sample of parameters values and using the collected data to construct a surrogate model for the constitutive law (by a simple interpolation).

A few remarks lead to a massive reduction of the cost of this characterization. First, the considered cells are orthotropic, hence only 9 coefficients (out of 36) of the homogenized tensor $A_{\omega_{0}}^{*}(m)$ have to be computed in order to characterize it. Second, the homogenized tensor does not have to be computed on the whole admissible set $[0,1]^{3}$ of the geometric parameters $m$. Indeed, a permutation $s$ of the parameters $m_{i}$ is equivalent to a rotation of $R\left(s\left(\omega_{0}\right)\right)$, where $s(\omega)=$ $\left(\omega_{s(1)}, \omega_{s(2)}, \omega_{s(3)}\right)$. Hence, we run the characterization of $A_{\omega_{0}}^{*}(m)$ only on the subset $\left\{m \in[0,1]^{3} \mid m_{1} \leq m_{2} \leq m_{3}\right\}$, dividing by a factor 6 the number of samples.

The computations of $A^{*}(m)$ are performed with FreeFem++ [17]. We used a regular discretization of parameters values, with 20 elements in each direction. The homogenized tensor is numerically represented as a $P 1$ function over the domain $[0,1]^{3}$. Hence the interpolation giving the homogenized tensor on the whole domain is automatically computed by FreeFem ++ . The Young modulus of the isotropic material used to design those cells is $E=15 G P a$ and its Poisson's ratio is $\nu=0.35$.

Results for $A^{*}(m)$ can not be easily displayed since it would involve 3 -d plots. Therefore we content ourselves in displaying only two slices of $A^{*}(m)$ in Figures 2(a) and 2(b). The parameter $m_{3}$ is fixed to 0.5 and the parameters $m_{1}$ and $m_{2}$ vary from zero to one. The coefficient $A_{1111}^{*}$ (respectively $A_{3333}^{*}$ ) and its gradient are displayed on Figure 2(a) (respectively 2(b)). Those coefficients are smooth functions of $m_{1}$ and $m_{2}$. Moreover they decrease when $m_{1}$ (respectively $m_{2}$ ) increases, as is expected. The entry $A_{1111}^{*}$ is more sensitive to a variation of $m_{2}$ when $m_{1}$ is fixed than the opposite. Indeed, the larger the $m_{2} \times m_{3}$ rectangular hole, the weaker the cell is in the direction $x_{1}$. Moreover, when $m_{2}=0$ (resp. $m_{1}=0$ ), the cell features a crack of normal $e_{2}$ (resp. $e_{1}$ ): the homogenized properties do not reach the ones of the full material. The entry $A_{3333}^{*}$ is a symmetric function of the parameters $m_{1}$ and $m_{2}$. Indeed, in the direction $x_{3}$, the more significant source of loss of rigidity comes from the $m_{1} \times m_{2}$ rectangular hole, no matter its orientation. When $m_{1}=m_{2}=0$, the cell features a crack line in the direction $x_{3}$. For a load in the same direction, the crack line has no impact: the homogenized coefficient $A_{3333}^{*}$ is equal to the same coefficient of the full material: $A_{3333}$. 


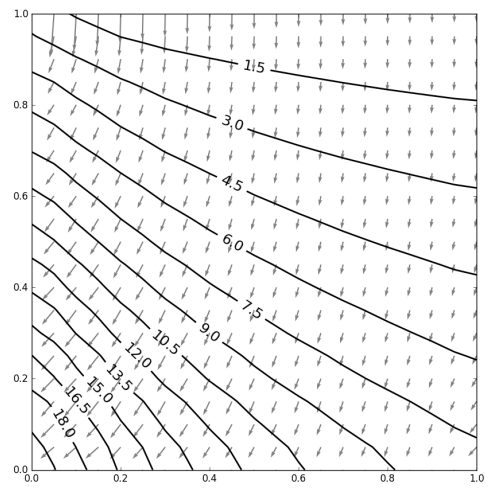

(a) $A_{1111}^{*}\left(m_{1}, m_{2}, 0.5\right)$ with $A_{1111}=25.58$

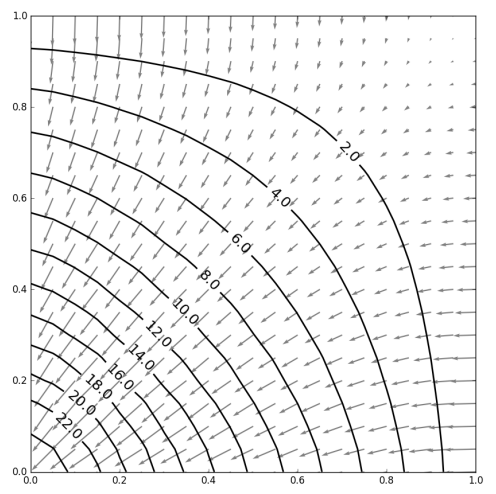

(b) $A_{3333}^{*}\left(m_{1}, m_{2}, 0.5\right)$ with $A_{3333}=25.58$

Figure 2: Isolines of the entries of the homogenized coefficients $A_{1111}^{*}$ (left) and $A_{3333}^{*}$ (right) and their gradients (small arrows) according to the parameters $\mathrm{m}$, with $m_{3}=0.5$. The $x$-axis is $m_{1}$, the $y$-axis is $m_{2}$

\section{Processing: optimization among the set of pe- riodic composite materials}

\subsection{Settings of the homogenized problem}

In this paper, only compliance minimization problems, as defined in Section 2, are considered. Recall that its relaxed version for composite designs amounts to minimize the cost function

$$
J^{*}\left(\theta, A^{*}\right)=\int_{\Gamma_{N}} g \cdot u^{*} d s,
$$

which is also equal to the minimum of complementary energy

$$
J^{*}\left(\theta, A^{*}\right)=\min _{\tau \in H_{0}} \int_{D} A^{*-1} \tau: \tau \mathrm{dx}
$$

where

$$
H_{0}=\left\{\begin{array}{ll} 
& \operatorname{div}(\tau)=0 \text { in } D \\
\tau \in L^{2}\left(D ; \mathcal{M}_{3}^{s}\right) \text { such that } & \tau n=g \text { on } \Gamma_{N} \\
& \tau n=0 \text { on } \Gamma
\end{array}\right\} .
$$

Thus, this compliance minimization problem can be recast as a double minimization problem over the stress field and the admissible microstructures [1]. 
Namely, it is equivalent to:

$$
\begin{aligned}
& \inf _{\substack{\sigma \in H_{0} \\
m \in L^{\infty}\left(D ;[0,1]^{2}\right)}} \int_{D} A_{\omega}^{*-1}(m) \sigma: \sigma \mathrm{dx}, \\
& \omega \in L^{\infty}(D ; W) \\
& \int_{D} \theta(m) \mathrm{dx} \leq V
\end{aligned}
$$

where

$$
\theta(m)=1-m_{1} m_{2}-m_{1} m_{3}-m_{2} m_{3}+2 m_{1} m_{2} m_{3}
$$

is the local density of the periodic microstructure of parameters $m$ and

$$
W=\left\{\left(\omega_{1}, \omega_{2}, \omega_{3}\right) \in\left(\mathbb{S}^{2}\right)^{3} \text { such that } \forall i, j \in\{1,2,3\} \quad \omega_{i} \cdot \omega_{j}=\delta_{i j}\right\}
$$

where $\mathbb{S}^{2}$ is the unit sphere in $\mathbb{R}^{3}$ and $\delta_{i j}$ is the Kronecker symbol.

In order to solve this problem, we use an alternate minimization algorithm [1], minimizing successively with respect to the stress field $\sigma$, the cell parameters $m$ and the orientation $\omega$ of the cell. We introduce the corresponding Lagrangian

$$
\mathcal{L}(m, \omega, \sigma, \ell)=\int_{D} A_{\omega}^{*-1}(m) \sigma: \sigma \mathrm{dx}+\ell\left(\int_{D} \theta(m) \mathrm{dx}-V\right),
$$

where $\ell$ is the Lagrange multiplier associated to the volume constraint.

To minimize with respect to the geometric parameters $m$, we use a projected gradient algorithm. Minimizing with respect to the stress field $\sigma$ amounts to solve the elasticity problem with a material of elasticity tensor equal to $A_{\omega}^{*}(m)$ in $D$. For more details on both minimizations, which are completely similar in 3 -d to the 2-case, we refer to our previous work [2]. However the minimization with respect to the orientation $\omega$ is different in $3-\mathrm{d}$, compared to $2-\mathrm{d}$, and is presented in full details.

Recall that there are various representations for 3-d orientation, like Euler angles or quaternions. They could be valid choices if a gradient descent method was used to compute the optimal orientation. However, this strategy may not be very efficient due to the occurrence of possible local minima. Besides, as proved by Pedersen [28] in 2-d and Norris [26] in 3-d, the optimal orientation of an orthotropic cell is given by the principal directions of the stress tensor. This result motivates our choice of modeling the orientation as the set of three unit vectors forming an orthonormal basis: $\omega=\left(\omega_{1}, \omega_{2}, \omega_{3}\right)$. Then the rotation matrix $Q(\omega)$ is simply the change-of-basis matrix from $\left(e_{1}, e_{2}, e_{3}\right)$ to $\left(\omega_{1}, \omega_{2}, \pm \omega_{3}\right)$, the sign \pm being chosen so that $\operatorname{det} Q(\omega)=1$ (see Section 3.3 for more details).

At each iteration $n$ of the optimization algorithm, the updated orientation $\omega_{n+1}(x)$ is then given by the three normalized (i.e. unit) eigenvectors of the stress tensor $\sigma_{n+1}(x)$. Moreover the vectors $\left(\omega_{1}, \omega_{2}, \omega_{3}\right)$ are labelled according to their associated eigenvalue (taken with their sign): from the smaller one to the larger one. Of course, unit eigenvectors are defined only up to their sign: they are not uniquely defined and only their direction is unambiguously defined. However, this sign ambiguity has no influence on the rotation operator $R(\omega)$, defined by (6). 
Remark 2. This approach is more efficient than the gradient descent method, mainly because it is a global minimization method, providing an optimal orientation at each iteration. However it works only for compliance minimization problem. For other objective functions, other methods should be implemented.

The complete optimization algorithm is the same as in 2-d [2]. It is an iterative method, structured as follows :

1. Initialization of the design parameters $(m, \omega)$, for example we take $m_{1}=$ $m_{2}=m_{3}$, constant satisfying the volume constraint, and $\omega=\omega_{0}=$ $\left(e_{1}, e_{2}, e_{3}\right)$.

2. Iteration until convergence, for $n \geq 0$ :

(a) Computation of the stress $\sigma^{n}$ through a problem of linear elasticity with $A_{\omega^{n}}^{*}\left(m^{n}\right)$ as elasticity tensor.

(b) Updating the orientation $\omega^{n+1}$, using Norris formula.

(c) Updating the cell parameters $m^{n+1}$ by one step of a projected gradient algorithm where the descent direction is given by the derivative according to $m$ of the Lagrangian (8) evaluated at $\sigma^{n}$ and $\omega^{n}$.

This topology optimization algorithm was implemented in the finite element software FreeFem $++[17]$ (see [3] for the use of FreeFem++ in optimal design). All unknown fields are discretized using $P 1$-functions.

At each iteration and for each node of the mesh, the principal directions of the stress tensor $\sigma_{n+1}$ are computed thanks to the library lapack. They are ordered according to their respective eigenvalue. In practice, Mandel notations are used, in order to replace tensors product by matrix product. Hence, the fourth-order tensor $R(\omega)$ is represented by a $6 \times 6$ matrix. Its expression in terms of $\omega$ is given in the appendix.

\subsection{Numerical results and discussion}

The previous optimization algorithm is discussed and illustrated for a cantilever problem, for which the boundary conditions are displayed on Figure 3. Other examples are performed in Section 6. The domain size is $15 \times 10 \times 5$ and it is discretized by a tetrahedral mesh with 3604 elements and 959 vertices. This mesh is coarse, a parallel implementation would enable the use of finer meshes. Decomposition domain methods [13] are available in FreeFem ++ , hence our algorithms could be parallelized with only few additional work. This has to be investigated.

The volume constraint is fixed at $35 \%$ of the working domain. The convergence history is displayed on Figure 4 : the algorithm converges smoothly and quickly.

The optimized density $\theta$ and cell parameter $m$ displayed on Figure 5. As usual, 3-d results are difficult to display clearly because only external borders are represented. Therefore, in Figure 6 various iso-surfaces of the density field 


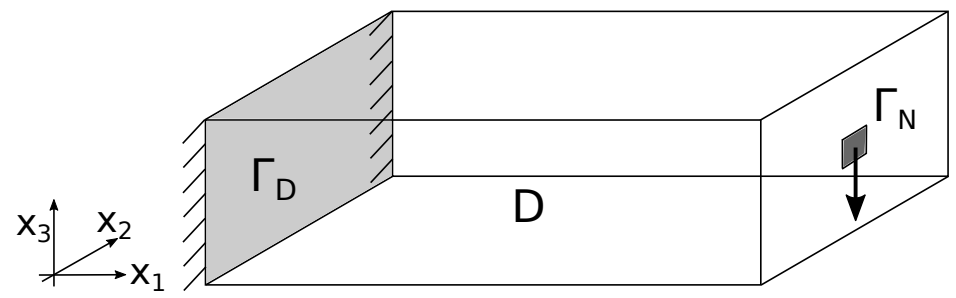

Figure 3: Boundary conditions for the cantilever problem

are plotted. The optimized structure is clearly not a black and white design. High densities are reached only around where Dirichlet and Neumann boundary conditions are applied. The major part of the domain is filled with intermediate or grey densities. Similarly, the optimized geometric parameters $\left(m_{1}, m_{2}, m_{3}\right)$ vary in their full range from 0 to 1 and they are not equal in a large region of the domain. The obtained optimal composite is clearly not isotropic. Moreover, the optimal orientation is displayed on Figures 7(a), 7(c) and 7(e). It is not constant in the whole domain : the obtained optimal composite takes advantage of the anisotropy of the cells.

For the sake of comparison, the same test case has been performed with different homogenized properties. Since minimal compliance for a single loadcase is known to be reached by rank-3 laminates [1], the optimal design for such laminates has also been computed. For the sake of brevity, the resulting design is not displayed here. The rank-3 laminate compliance is equal to 14.802 and is indeed lower than the compliance reached by our periodic optimal homogenized structure, which is 20.933. Such a large gap in performance is obviously due to our constraint of choosing a periodic cell with connected holes (see Figure 1) for manufacturability reasons, while the rank-3 laminates feature closed holes which are definitely better for structural performance. Another comparison is made with the Solid Isotropic Material with Penalization (SIMP) method, including a sensitivity filter (in order to avoid checkerboards). The homogenized tensor $A^{*}$ is replaced by $\theta^{p} A$ where $A$ is the pure material Hooke's law and the maximal exponent is $p=3$ in the power law of the density. The result is displayed in Figure 8 since it is a black and white design, more suitable to be displayed. The optimal compliance reached by this method is 22.401 , which is $7 \%$ greater than the compliance reached by the optimal periodic homogenized structure. The discretized mesh was the same for all test cases, as well as the ersatz material ( $0.1 \%$ of the elastic tensor of the isotropic material).

\subsection{Regularization of the orientation}

The orientation might be not very smooth in some regions, in particular in the areas of either full or zero density, in which the material is isotropic. It is possible to regularize the orientation field without changing too much the performance of the final structure. The interest in regularizing the orientation is crucial for 


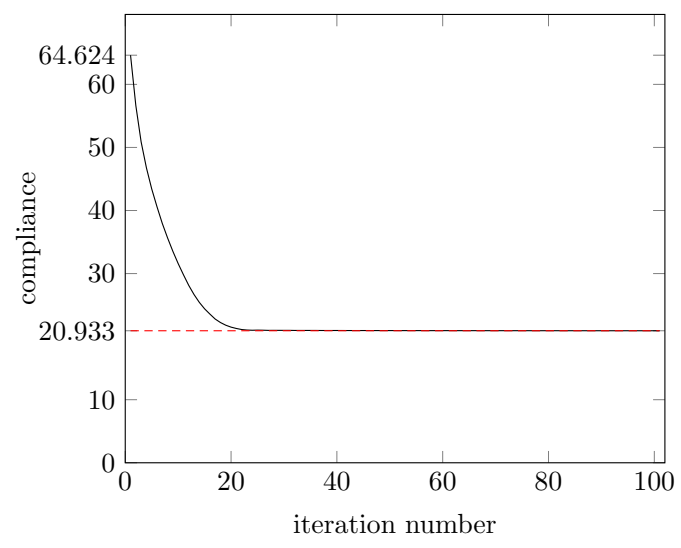

Figure 4: Convergence history of the objective function (compliance) for the bridge

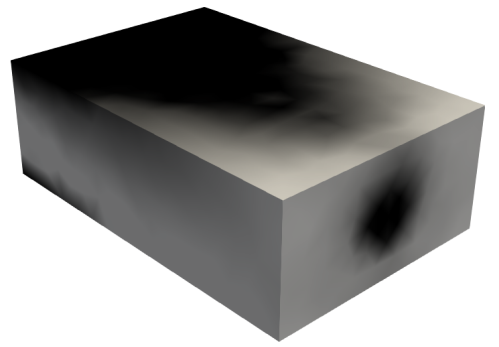

(a) $m_{1}$

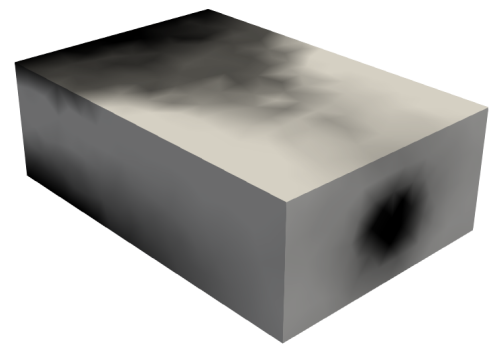

(c) $m_{3}$

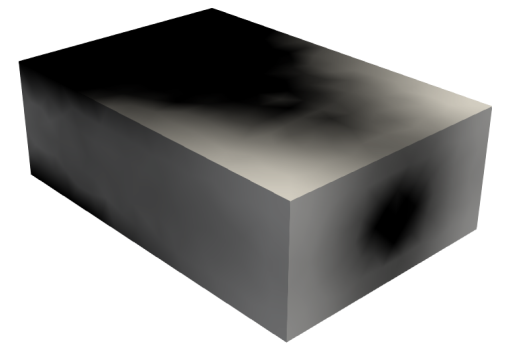

(b) $m_{2}$

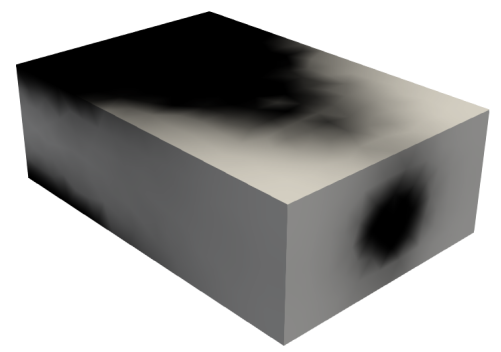

(d) density

Figure 5: Optimized geometric parameters $m$ and density of the cantilever

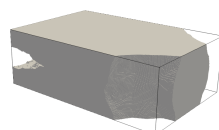

(a) $\theta \geq 10 \%$

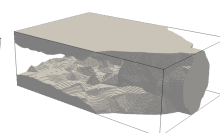

(b) $\theta \geq 25 \%$

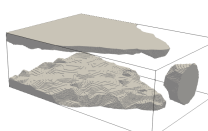

(c) $\theta \geq 50 \%$

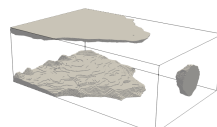

(d) $\theta \geq 75 \%$

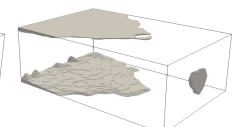

(e) $\theta \geq 90 \%$

Figure 6: Threshold of the domain according to the optimized density $\theta$ for the cantilever case 


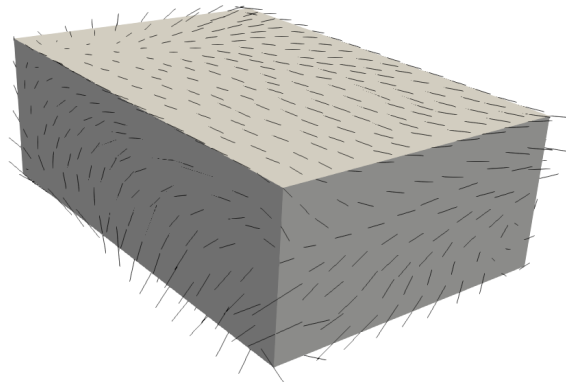

(a) Optimized $\omega_{1}$

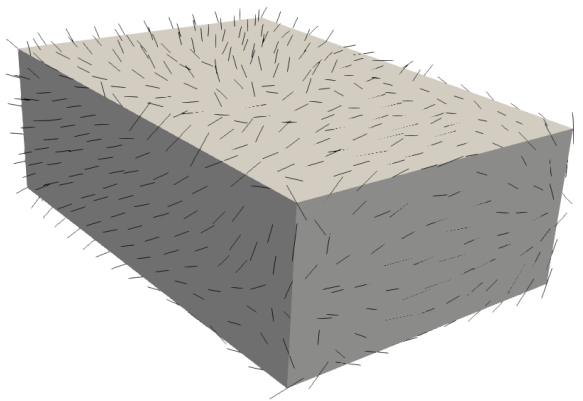

(c) Optimized $\omega_{2}$

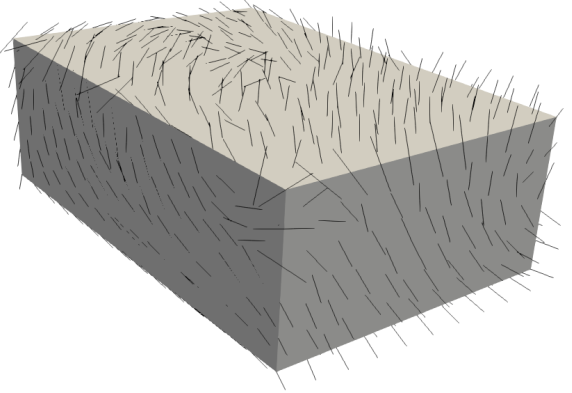

(e) Optimized $\omega_{3}$

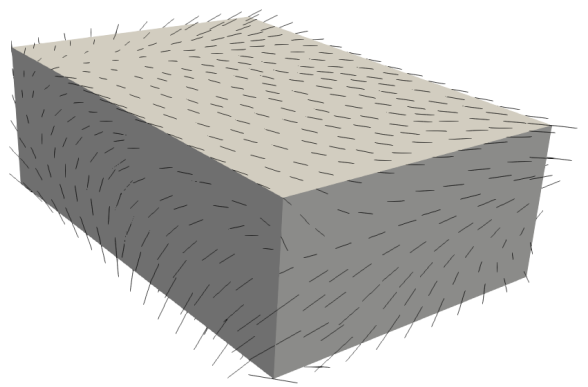

(b) Regularized $\omega_{1}$

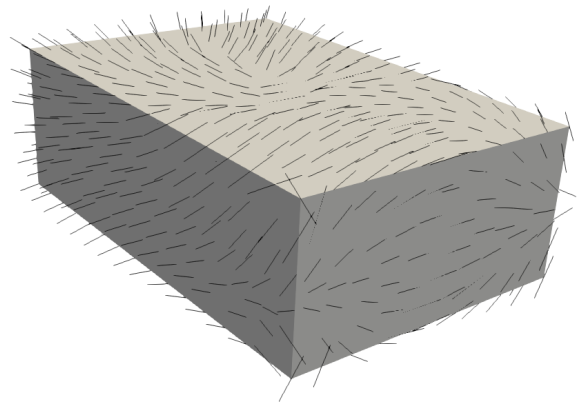

(d) Regularized $\omega_{2}$

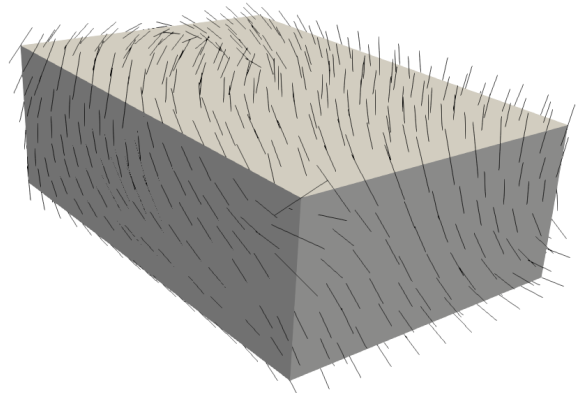

(f) Regularized $\omega_{3}$

Figure 7: Optimized (left) and regularized (right) orientation for the cantilever case

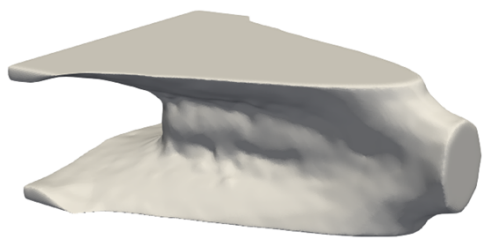

Figure 8: Optimized design of the cantilever with penalized SIMP method 
the post-processing stage to lead to reasonable results. The orientation in those black and white areas has no influence on the performances of the homogenized structure. However, they may sensibly degrade the computation of the grid map $\varphi$, which distorts a regular grid in order to align the cells along a given local orientation, see Section 5.

Classically, in order to regularize the orientation, we would like to minimize a function:

$$
\sum_{i=1}^{3}\left\|\nabla \omega_{i}\right\|_{L^{2}(D)}^{2}
$$

where $\|\cdot\|_{L^{2}(D)}^{2}$ denotes the integral over $D$ of the (squared) Frobenius norm (the same notation shall be used for tensors of order 3 and 4 too).

However, since the orientation vectors $\omega_{i}$ are the principal directions of the stress tensor, they are defined locally up to their sign and can feature discontinuities. Then, there is no hope to compute directly their gradient. A possible approach would be to introduce a manifold, like in [2] in order to evaluate the gradients. This approach is doable but since we use an iterative algorithm in order to regularize the orientation, we should recompute interpolation operators at each iteration, which is too time-consuming.

Regularized cost function. We present here another approach, based on the fact that the $\omega_{i}$ are computed as the principal directions of a symmetric matrix. The main idea amounts not to regularize directly the orientation $\omega$ but rather a symmetric matrix $M(\omega)$, independent of the arbitrary chosen sign for the orientation vectors $\omega_{i}$. Recall that $Q(\omega)$ is the rotation matrix from $\left(e_{1}, e_{2}, e_{3}\right)$ to $\left(\omega_{1}, \omega_{2}, \pm \omega_{3}\right)$. Let $\mathcal{D}(\lambda)$ be the diagonal matrix, with entries $\lambda_{1}=-1, \lambda_{2}=1$ and $\lambda_{3}=0$. We define a symmetric matrix $M$ by

$$
M(\omega)=Q(\omega)^{T} \mathcal{D}(\lambda) Q(\omega)
$$

whose eigenvalues are $\lambda_{i}$ and associated eigenvectors are $\omega_{i}$. Since we want to regularize the orientation without degrading the compliance, we introduce a new cost-function to minimize:

$$
J_{\mathrm{reg}}(m, \omega)=\int_{D} A_{\omega}^{*-1}(m) \sigma: \sigma d x+\eta_{\mathrm{reg}}^{2}\|\nabla M(\omega)\|_{L^{2}(D)}^{2},
$$

with $\sigma$ the stress tensor solution of the elasticity problem and $\eta_{\text {reg }}>0$, a small coefficient. In practice, $\eta_{\mathrm{reg}}=0.1$ works well.

Approximate minimization. We now describe a SQP type algorithm to minimize this cost function $J_{\text {reg }}$ with respect to $\omega$. The optimization process with respect to $m$ is unchanged. At each iteration $n$, we approximate $J_{\text {reg }}$ at second order in $\delta \omega$, around the current state, by

$J_{\operatorname{approx}}(m, \omega+\delta \omega)=J_{\operatorname{reg}}(m, \omega)+<J_{\operatorname{reg}}^{\prime}(m, \omega), \delta \omega>+\frac{1}{2}<J_{\operatorname{reg}}^{\prime \prime}(m, \omega) \delta \omega, \delta \omega>$. 
In theory, $\omega+\delta \omega$ should belong to $W$, defined by (7). Since the sign of the vectors of the orientation does not matter, we can impose without loss of generality that $\omega$ and $\omega+\delta \omega$ have the same determinant. In other words, $\omega$ and $\omega+\delta \omega$ are both either direct triads or indirect triads and we have:

$$
\forall i \in\{1,2,3\} \quad \omega_{i}+\delta \omega_{i}=S \omega_{i}, \quad \text { where } \quad S=Q(\omega+\delta \omega) Q(\omega)^{T} \in S O(3),
$$

where $S O(3)$ is the special orthogonal group. First, we change the unknown,

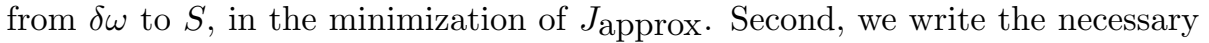
and sufficient optimality condition of this minimization problem as the variational problem consisting in finding $S^{n} \in H^{1}(D, S O(3))$ such that for all test functions $T \in H^{1}(D, S O(3))$ :

$$
\begin{aligned}
& <J_{\text {reg }}^{\prime}\left(m, \omega^{n}\right),\left(\left(T-I d_{3}\right) \omega^{n}\right)> \\
+\quad & <J_{\text {reg }}^{\prime \prime}\left(m, \omega^{n}\right)\left(\left(S^{n}-I d_{3}\right) \omega^{n}\right),\left(\left(T-I d_{3}\right) \omega^{n}\right)>=0
\end{aligned}
$$

The orientation is then updated with:

$$
\omega^{n+1}=S^{n} \omega^{n} .
$$

Nevertheless, problem (12) is still too complex. So, we approximate it by another variational problem whose unknown is no longer a field of orthogonal matrices in $S O(3)$, but a field of vectors in $\mathbb{R}^{3}$. This approximation is based on the following idea: since an iterative method is used for regularizing the orientation, between two iterations the orientation should not change drastically. This means that the rotation matrix $S^{n}$ should be close to the identity $I d_{3}$. Hence the variational formulation (12) is approximated in a neighbourhood of $I d_{3}$, which is not a subset of $S O(3)$ (the numerical approximation of a neighbourhood of $I d_{3}$ in $S O(3)$ is too complicated). In what follows we explain how to construct this neighbourhood. First, the set of admissible rotation matrices $S$ in the variational problem (12) is parametrized. Second, this parametrization allows us to build a neighborhood of $I d_{3}$.

Since the unknown matrix $S^{n}$ in (12) is close to the identity, -1 is not one of its eigenvalues. The Cayley transform ensures there exists an involution between the set of skew-symmetric matrices and the set of orthogonal matrix, for which no eigenvalue is equal to -1 . Hence, the set of admissible rotation matrices $S O(3)$ in the variational problem (12) can be restricted to:

$$
\left\{\left(I d_{3}-N\right)^{-1}\left(I d_{3}+N\right) \quad \mid \quad N \in \mathcal{M}_{3}(\mathbb{R}) \text { such that } N^{T}=-N\right\} .
$$

Using again that $S^{n}$ is close to the identity $I d_{3}$, by an asymptotic expansion, we approximate (13) by the following neighbourhood of $I d_{3}$

$$
\mathcal{V}_{I d}=\left\{I d_{3}+2 N \quad \mid \quad N \in \mathcal{M}_{3}(\mathbb{R}) \text { such that } N^{T}=-N \text { and }\|N\|<<1\right\} .
$$

Skew matrices in $\mathcal{M}_{3}(\mathbb{R})$ can be parametrized by a vector in $\delta s \in \mathbb{R}^{3}$ as follows

$$
F(\delta s)=\left(\begin{array}{ccc}
0 & -\delta s_{3} & -\delta s_{2} \\
\delta s_{3} & 0 & -\delta s_{1} \\
\delta s_{2} & \delta s_{1} & 0
\end{array}\right)
$$


Then (14) can be rewritten as:

$$
\mathcal{V}_{I d}=\left\{I d_{3}+F(\delta s) \quad \mid \quad \delta s \in H^{1}\left(D, \mathbb{R}^{3}\right) \quad \text { such that } \quad\|\delta s\|<<1\right\} .
$$

Under the hypothesis that $S^{n}$ is close to $I d_{3}$, the variational problem (12) is approximated by a variational problem where $H^{1}(D, S O(3))$ is replaced by $\mathcal{V}_{I d}$. We take this opportunity to further add a penalization term on the norm of $\delta s$ in order to ensure that $\delta s$ is indeed small. The final approximate variational formulation is: find $\delta s^{n} \in H^{1}\left(D, \mathbb{R}^{3}\right)$ such that, for any test function $\delta \tau \in$ $H^{1}\left(D, \mathbb{R}^{3}\right)$,

$$
\begin{aligned}
& <J_{\text {reg }}^{\prime}\left(m, \omega^{n}\right),\left(F(\delta \tau) \omega^{n}\right)> \\
+\quad & <J_{\text {reg }}^{\prime \prime}\left(m, \omega^{n}\right)\left(F\left(\delta s^{n}\right) \omega^{n}\right),\left(F(\delta \tau) \omega^{n}\right)> \\
+ & \frac{1}{\eta_{\text {pen }}^{2}}<\delta s^{n}, \delta \tau>=0,
\end{aligned}
$$

where $\eta_{\text {pen }}>0$ is a coefficient, small enough to ensure the stability of the gradient algorithm. In practice, we take $\eta_{\text {pen }}=1$.

Finally, to update the orientation, we compute the matrix:

$$
S^{n}=I d_{3}+F\left(\delta s^{n}\right),
$$

which is not a rotation matrix, as already said. Hence $\omega^{n+1}$ is not equal to $S^{n} \omega^{n}$ but rather is given by a Gram-Schmidt orthonormalization process, applied to the set of vectors $\left(S^{n} \omega_{1}^{n}, S^{n} \omega_{2}^{n}, S^{n} \omega_{3}^{n}\right)$. Several approximations have been used, and their combination is not guaranteed to work well. However, in practice the algorithm converges and its implementation is rather simple.

Derivatives of $J_{\text {reg. }}$ To make (15) fully explicit, we now give formulas for the derivatives of $J_{\mathrm{reg}}(m, \omega)$ with respect to $\omega$. From the definitions (9) of $M$ and (11) of $S$, we get:

$$
M(\omega+\delta \omega)=M(\omega)+\left(F^{T}(\delta s) M(\omega)+M(\omega) F(\delta s)\right)+F(\delta s) M(\omega) F(\delta s)^{T} .
$$

Eventually, it leads to

$$
\begin{array}{r}
<J_{\mathrm{reg}}^{\prime}(m, \omega),(F(\delta \tau) \delta \omega)>=\int_{D}\left(A_{\omega_{0}}^{*-1}(m) R(\omega) \sigma\right):\left(R^{\prime}(F(\delta \tau) \omega) \sigma\right) d x \\
+\eta_{\mathrm{reg}}^{2} \sum_{1 \leq i, j \leq 3} \int_{D} 2 \nabla M_{i, j}(\omega) \cdot \nabla\left(F^{T}(\delta \tau) M+M F(\delta \tau)\right)_{i, j} d x,
\end{array}
$$

and

$$
\begin{aligned}
& <J_{\text {reg }}^{\prime \prime}(m, \omega)(F(\delta \tau) \delta \omega),(F(\delta s) \delta \omega)>=2 \int_{D} A_{\omega_{0}}^{*-1}(m) R^{\prime}(F(\delta s) \omega) \sigma: R^{\prime}(F(\delta \tau) \omega) \sigma d x \\
& +2 \eta_{\mathrm{reg}}^{2} \sum_{1 \leq i, j \leq 3} \int_{D} 2 \nabla M_{i, j}(\omega) \cdot \nabla\left(F^{T}(\delta s) M F(\delta \tau)\right)_{i, j} d x \\
& \left.+2 \eta_{\mathrm{reg}}^{2} \sum_{1 \leq i, j \leq 3} \int_{D} \nabla\left(F^{T}(\delta s)\right) M+M F(\delta s)\right)_{i, j} \cdot \nabla\left(F^{T}(\delta \tau) M+M F(\delta \tau)\right)_{i, j} d x,
\end{aligned}
$$




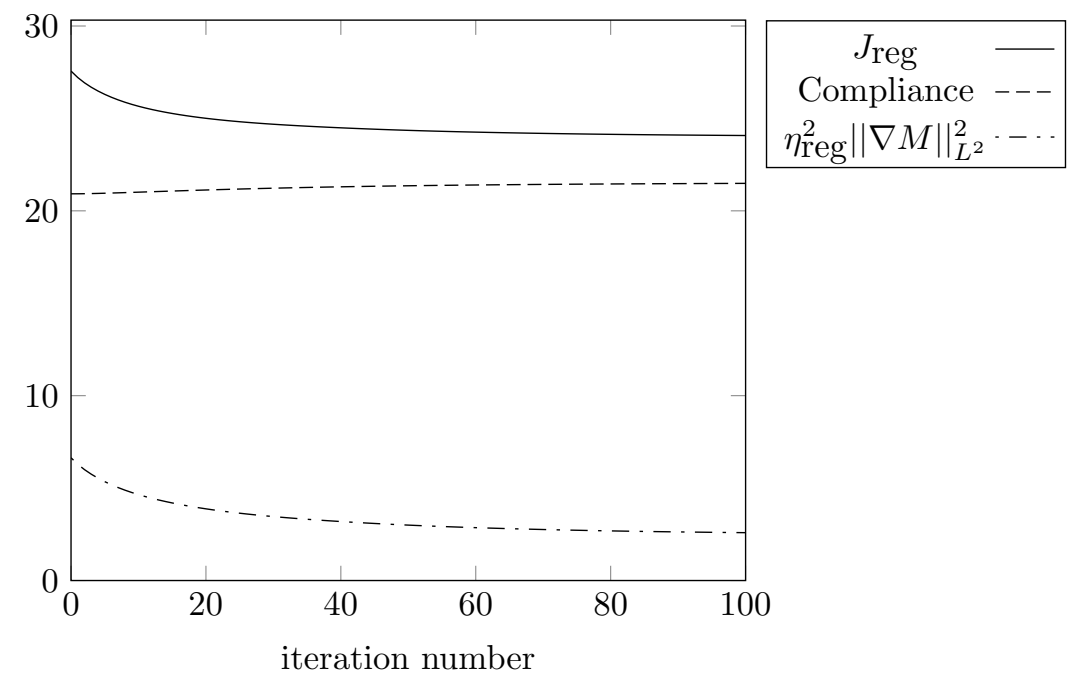

Figure 9: Convergence history of the regularization cost function $J_{\text {reg }}$ for the bridge

where $R^{\prime}(v)$ is the directional derivative of $R(\omega)$ in the direction $v$, and $A_{\omega_{0}}^{*}$ is the homogenized elasticity tensor in the reference orientation $\omega_{0}$.

Numerical results. The above algorithm has been implemented in FreeFem ++ , using again $P 1$ functions for all the unknown fields. Recall that $\lambda_{1}=-1, \lambda_{2}=1$ and $\lambda_{3}=0$. Hence the three vector fields $\omega_{i}$ are equally regularized. Indeed $\omega_{1}$ and $\omega_{2}$ have the same regularization coefficient since $\left|\lambda_{1}\right|=\left|\lambda_{2}\right|$, and the last one is fully determined by the others thanks to the orthogonality of the eigenvectors for a symmetric matrix. For the cantilever case, the regularized vector fields are displayed on Figures $7(\mathrm{~b}), 7(\mathrm{~d})$ and $7(\mathrm{f})$. The orientation vectors are not changed much by the regularization step, except in the black and white design regions. As can be checked in Figure 9, during the regularization process, the compliance is only slightly increasing while the regularization term undergoes a strict decrease.

\section{Post-Processing : projection of the optimized microstructure}

The last step is to construct a sequence of classical or genuine shapes that approximates the computed optimal composite. This sequence is indexed by a small positive parameter $\varepsilon>0$ which is the characteristic size of the period of the periodic composite. In practice, the parameter $\varepsilon$ is set to a value freely chosen by the user. Of course, the smaller $\varepsilon$, the more detailed will be the resulting genuine shape. 
The main ideas are roughly the same as in 2-d [2] except for the following crucial points. In 2-d, we took advantage of conformal maps in 2-d in order to compute a distortion map. We can no longer make this assumption in 3-d and we present a different approach here. Moreover, the treatment of the orientation in 3-d is quite different as in 2-d, which implies to adapt our algorithms. The fact that the distortion map is not a conformal map implies that the sequence of genuine shapes does not converge exactly to the computed optimal composite, but only to a close approximation.

\subsection{Sequences of shapes}

Similarly to the 2-d case, we introduce a map $\varphi=\left(\varphi_{1}, \varphi_{2}, \varphi_{3}\right)$ from $D$ into $\mathbb{R}^{3}$, in order to properly orient each cell locally, without connectivity failures in the final structure. This map distorts a regular grid, such that each bar in direction $e_{i}$ is locally oriented along $\omega_{i}$.

For all $i \in\{1,2,3\}$, let $Y_{i}$ be defined by:

$$
Y_{i}(m)=\left\{y \in[0,1]^{3} \text { such that } \cos \left(2 \pi y_{i}\right) \geq \cos \left(\pi\left(1-m_{i}\right)\right)\right\} .
$$

$Y_{i}$ is the unit cube, cut by a central slice of normal $e_{i}$, and thickness $m_{i}$, see Figure 10. Our unit cells $Y_{0}(m)$ can then be analytically defined by:

$$
Y_{0}(m)=\cup_{1 \leq i<j \leq 3}\left(Y_{i}(m) \cap Y_{j}(m)\right)
$$

In order to compute a sequence of genuine shapes approximating the optimized homogenized structures, we introduce $\varepsilon$ the reference size of the cells, which is going to 0 . The elements of this sequence are denoted by $\Omega_{\varphi, \varepsilon}(m)$, since they depend on the map $\varphi$ that gives the proper orientation. We give details about its computation further.

In periodic homogenization, we have two systems of coordinates : $x$, the macroscopic coordinates, and $y$ the microscopic coordinates, indicating the position into the periodic cell. Classically, we take $y=\frac{x}{\epsilon}-E\left(\frac{x}{\epsilon}\right)$ to describe a homogenized periodic structure, where $E(\cdot)$ is the floor function.

Thanks to the map $\varphi$, that distorts the regular grid, the new macroscopic coordinates to be considered are then $\varphi(x)$. The sequence of genuine shapes is then built according to the exact same scheme as the unit cell $Y_{0}(m)$. Let us introduce the counterparts of $Y_{i}(m)$ :

$$
\Omega_{i, \varphi, \varepsilon}(m)=\left\{x \in D \text { such that } \cos \left(\frac{2 \pi \varphi_{i}\left(x_{i}\right)}{\epsilon}\right) \geq \cos \left(\frac{\pi\left(1-m_{i}\right)}{\epsilon}\right)\right\} .
$$

Hence, the sequence of genuine shapes is given by:

$$
\Omega_{\varphi, \varepsilon}(m)=\cup_{1 \leq i<j \leq 3}\left(\Omega_{i, \varphi, \varepsilon}(m) \cap \Omega_{j, \varphi, \varepsilon}(m)\right)
$$

We emphasize that thanks to the periodicity of the cosine function, there is no need to consider the integer part of $\frac{x}{\epsilon}$ in the approximation of the microscopic coordinates. 

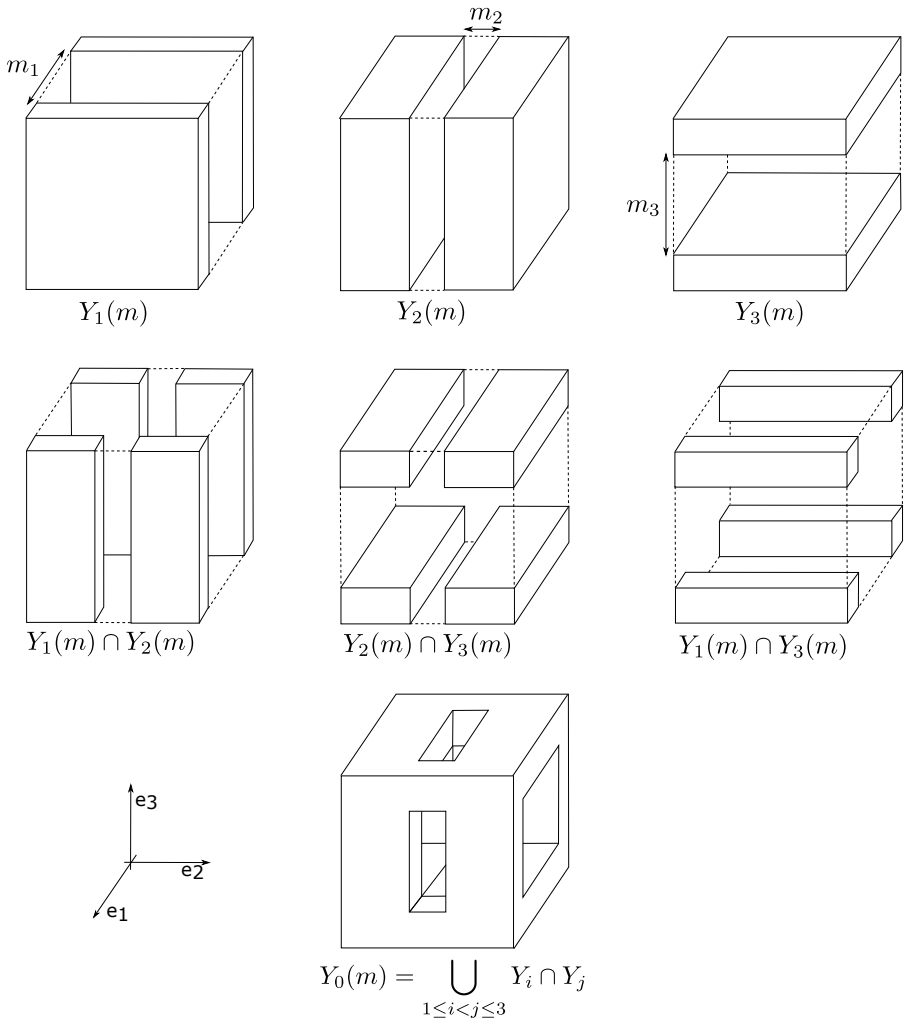

Figure 10: Construction of the cell $Y_{0}(m)$ from $Y_{i}(m)$.

Remark 3. The map $\varphi$ is introduced in order to take into account the optimal orientation of the cell. Hence, $\varphi$ depends only on the orientation $\omega$. In particular, $\varphi$ does not depend on the size $\varepsilon$ of the cells. Once $\varphi$ has been computed, it could be used for any value of this scale parameter.

\subsection{Computation of the projection map $\varphi$}

We are looking for a map $\varphi=\left(\varphi_{1}, \varphi_{2}, \varphi_{3}\right)$ from $D$ into $\mathbb{R}^{3}$ such that its gradients $\nabla \varphi_{i}$ are aligned with $\omega_{i}$. Hence, we impose for the map $\varphi$ to satisfy:

$$
\forall i \in\{1,2,3\} \quad \nabla \varphi_{i}=e^{r_{i}} \omega_{i}
$$

where $r_{i}$ is a scalar field, given the local dilation of the cubic cell in the direction $\omega_{i}$.

Unlike the 2-d case, we can not assume here that the three fields $r_{i}$ are the same field. Indeed, if the values of $r_{i}$ are locally equal to each other, infinitesimal cubes is distorted into cubes. If the fields $r_{i}$ were equal everywhere to each 
other, then the map $\varphi$ would be a conformal map. However, there is only a few conformal maps in 3-d, thanks to Liouville's theorem [29]. Hence, it would be too restrictive to impose such a condition on the map $\varphi$.

Taking the rotational of (20) leads to:

$$
\forall i \in\{1,2,3\} \quad\left(\nabla r_{i} \wedge \omega_{i}+\nabla \wedge \omega_{i}\right)=0
$$

Those equations do not define uniquely the dilation factors $r_{i}$ : they give the gradient of $r_{i}$ in only two directions, the orthogonal ones to $\omega_{i}$. We then penalize the third component by minimizing :

$$
\forall i \in\{1,2,3\} \quad \min _{s_{i}} \int_{D}\left|\nabla s_{i} \wedge \omega_{i}+\nabla \wedge \omega_{i}\right|^{2}+\eta_{1}^{2}\left|\nabla s_{i} \cdot \omega_{i}\right|^{2} .
$$

Hence, the dilations factors are defined up to a constant, that we can fix, by small penalization of the $L^{2}$ norm of $r_{i}$.

$$
\forall i \in\{1,2,3\} \quad \min _{s_{i}} \int_{D}\left|\nabla s_{i} \wedge \omega_{i}+\nabla \wedge \omega_{i}\right|^{2}+\eta_{1}^{2}\left|\nabla s_{i} \cdot \omega_{i}\right|^{2}+\eta_{2}^{2}\left|s_{i}\right|^{2} .
$$

The minimizer $\widehat{r}_{i}$ is the solution of the variational formulation consisting in finding $\widehat{r}_{i} \in H^{1}(D, \mathbb{R})$ such that for all $t \in H^{1}(D, \mathbb{R})$ :

$$
\int_{D}\left(\nabla \widehat{r}_{i} \wedge \omega_{i}+\nabla \wedge \omega_{i}\right) \cdot\left(\nabla t \wedge \omega_{i}\right)+\eta_{1}^{2}\left(\nabla \widehat{r}_{i} \cdot \omega_{i}\right)\left(\nabla t \cdot \omega_{i}\right)+\eta_{2}^{2} \widehat{r}_{i} t=0 .
$$

We emphasize that the solution $\widehat{r}_{i}$ of the above variational formulation does not verify the equation (21). Indeed, $\widehat{r}_{i}$ is an approximation of a theoretically perfect dilation factor $r_{i}$. In the following, we will no longer make the distinction between them, and we will indifferently write $r_{i}$.

Once the dilation factors $r_{i}$ are computed for each direction, the computation of the distortion map is reduced to the following minimization problem:

$$
\min _{\psi} \sum_{i=1}^{3} \int_{D}\left|\nabla \psi-e^{r_{i}} \omega_{i}\right|^{2}
$$

which can be separated in three independent minimization problems over the three components of $\varphi$. The minimizers $\varphi_{i}$ are respectively the solutions of the variational problems consisting in finding $\varphi_{i} \in H^{1}(D, \mathbb{R})$ such that for all $\psi \in H^{1}(D, \mathbb{R})$ :

$$
\int_{D}\left(\nabla \varphi_{i}-e^{r_{i}} \omega_{i}\right) \cdot \nabla \psi=0
$$

\subsection{Coherent orientation}

We compute separately the three components $\varphi_{i}$ of the map $\varphi$. First, we have to compute the dilation factor $r_{i}$. We see from the variational formulation (24) 
over the dilation factor that it depends on only one of the orientation vectors, namely $\omega_{i}$. Likewise, we can see from the variational formulation (26) over $\varphi_{i}$, that only the orientation vector $\omega_{i}$ is involved. Hence, we can consider each vector field separately.

We recall here that the representation of the optimal orientation using the vectors $\omega$. is not unique, since it is independent of their sign.

Numerically, we only compute the principal directions of the stress tensors, without any constraint about the regularity of the fields $\omega_{i}$. It might features discontinuities, coming from an uncontrolled change of sign. Because of their sign indetermination, the rotational of the vectors $\omega_{i}$, like in the above variational formulation (24) can not be directly computed.

A first approach amounts to reorientate each vector field by looking over the mesh, which is not efficient in the presence of singularities in the orientation field. Therefore, we prefer a second approach, similar to the one developed in [2]. We introduce a manifold, defined as a two-fold covering space of the working domain. On each of those subsets the orientation vector fields are coherent and have opposed signs. Defining an atlas over those manifolds, we are now able to address this sign problem. We will give more details in the following. We do not consider the possible presence of singularities in the orientation $\omega$ in the present work.

For each $i$, we introduce a covering space of the working domain $D$ :

$$
\mathcal{D}_{i}=\left\{(x, T) \in D \times \mathbb{S}^{2} \text { such that } T \wedge \omega_{i}(x)=0\right\},
$$

where $\mathbb{S}^{2}$ is the unit sphere in $\mathbb{R}^{3}$ (in other words, $\mathcal{D}_{i}$ is a submanifold of $D \times \mathbb{S}^{2}$ ). Let $\mathcal{U}$ be a covering of $D$ by open sets $U$. Since we assume that the optimal orientation does not feature any singularity, for each open set $U$ there exists a continuous map $T_{U, i} \in \mathcal{C}\left(U, \mathbb{S}^{2}\right)$ such that for all $x \in U, T_{U, i}(x)= \pm \omega_{i}(x)$. Two charts $g_{U, i}^{+}$and $g_{U, i}^{-}$of $\mathcal{D}_{i}$ are then defined:

$$
g_{U, i}^{+}: \begin{aligned}
U & \rightarrow \mathcal{D}_{i} \\
x & \mapsto\left(x, T_{U, i}(x)\right)
\end{aligned} \quad \text { and } \quad g_{U, i}^{-}: \begin{aligned}
U & \rightarrow \mathcal{D}_{i} \\
x & \mapsto\left(x,-T_{U, i}(x)\right)
\end{aligned}
$$

If the domain $D$ is simply connected and $\omega_{i}$ does not feature any singularity, the manifold $\mathcal{D}_{i}$ consists of two disconnected copies of the working domain $D$, on which the vector field $\omega_{i}$ is coherently oriented. Those two copies are only distinguished by the sign of $T$.

Integrals on the manifold $\mathcal{D}_{i}$. We introduce here the notion of integrals on the manifold $\mathcal{D}_{i}$.

Let $\mathcal{U}$ be a finite family of disjoint open subsets $U$ covering $D$, and $f \in$ $\mathcal{C}\left(\mathcal{D}_{i}, \mathbb{R}\right)$, a continuous map. We define the integral of $f$ over $\mathcal{D}_{i}$ by:

$$
\int_{D_{i}} f(x, T) d x=\sum_{U \in \mathcal{U}} \int_{U} f \circ g_{U, i}^{+}(x) d x+\int_{U} f \circ g_{U, i}^{-}(x) d x,
$$


where the charts $g_{U, i}^{ \pm}$are defined by (27). This definition does not depend on the family $\mathcal{U}$ of disjoint subsets.

Differential operators on the manifold $\mathcal{D}_{i}$. The manifold $\mathcal{D}_{i}$ can be endowed with a differential structure: a gradient operator and a rotational operator are induced on $\mathcal{D}_{i}$ as follows.

Let $x \in D$ and $U$ be an open subset of $D$ containing $x$, we define the gradient operator by:

$$
\nabla f(x, T)=\nabla\left(f \circ g_{U, i}\right)(x),
$$

where $f \in H^{1}\left(\mathcal{D}_{i}, \mathbb{R}\right)$ and $g_{U, i}$ is a chart of the neighborhood of $(x, T)$, and the rotational operator by:

$$
\nabla \wedge W(x, T)=\nabla \wedge\left(W \circ g_{U, i}\right)(x),
$$

where $W \in H^{1}\left(\mathcal{D}_{i}, \mathbb{R}^{3}\right)$.

Those operators do not depend of the choice of the open subset $U$.

Symmetric and antisymmetric maps on the manifold $\mathcal{D}_{i}$. We introduce the subset of symmetric maps on $\mathcal{D}_{i}$ :

$$
\mathcal{V}_{i}^{s}=\left\{f \in H^{1}\left(\mathcal{D}_{i}, \mathbb{R}\right) \text { such that: } \forall(x, T) \in \mathcal{D}_{i} \quad f(x,-T)=f(x, T)\right\}
$$

and of antisymmetric maps on $\mathcal{D}_{i}$ :

$$
\mathcal{V}_{i}^{a}=\left\{f \in H^{1}\left(\mathcal{D}_{i}, \mathbb{R}\right) \text { such that: } \forall(x, T) \in \mathcal{D}_{i} \quad f(x,-T)=-f(x, T)\right\} .
$$

Remark 4. The integral of a symmetric map over the manifold $\mathcal{D}_{i}$ can be simply rewritten. Let $\mathcal{U}$ be a finite family of disjoint open subsets $U$ covering $D$, and $f \in \mathcal{V}_{i}^{s}$. We have:

$$
\begin{aligned}
\int_{D_{i}} f(x, T) d x & =\sum_{U \in \mathcal{U}}\left(\int_{U} f \circ g_{U, i}^{+}(x) d x+\int_{U} f \circ g_{U, i}^{-}(x) d x\right) \\
& =2 \sum_{U \in \mathcal{U}}\left(\int_{U} f \circ g_{U, i}^{+}(x) d x\right) .
\end{aligned}
$$

We now have all the requirements to change our working space from $D$ to $\mathcal{D}_{i}$ : we are looking for a dilation map $r_{i}$ from $\mathcal{D}_{i}$ into $\mathbb{R}$ and a grid map $\varphi_{i}$ from $\mathcal{D}_{i}$ into $\mathbb{R}^{3}$.

Dilation map $r_{i}$. The dilation map $r_{i}$ satisfied (21) on $D$. Therefore it also satisfies on $\mathcal{D}_{i}$ :

$$
\nabla r_{i} \wedge T+\nabla \wedge T=0
$$

A solution of the above equation is also solution of the same equation where $-T$ replaces $T$. We emphasize that there is no unicity of the solution, since we can create a new solution from a previous one simply by adding a constant to it. Then, we assume that $r_{i}$ satisfies a symmetric property on $\mathcal{D}_{i}$, meaning that $r_{i} \in \mathcal{V}_{i}^{s}$, without loss of generality. Hence, from (23), we compute $r_{i}$ as the minimizer of:

$$
\min _{s_{i} \in \mathcal{V}_{i}^{s}} \int_{\mathcal{D}_{i}}\left|\nabla s_{i} \wedge T+\nabla \wedge T\right|^{2}+\eta^{2}\left|\nabla s_{i} \cdot T\right|^{2}+\eta_{N}^{2}\left|s_{i}\right|^{2} d x
$$


Grid map $\varphi_{i}$. The grid map $\varphi_{i}$ satisfies on $\mathcal{D}_{i}$ :

$$
\nabla \varphi_{i}=e^{r_{i}} T
$$

The opposite of a solution of the above equation is also solution of the same equation where $-T$ replaces $T$. As previously for the dilation maps, we emphasize there is no unicity of the solution. We assume that $\varphi_{i}$ satisfies an antisymmetric property on $\mathcal{D}_{i}$, meaning that $\varphi_{i} \in \mathcal{V}_{i}^{a}$, without loss of generality. Hence, $\varphi_{i}$ is the minimizer of:

$$
\min _{\psi_{i} \in \mathcal{V}_{i}^{a}} \int_{\mathcal{D}_{i}}\left|\nabla \psi_{i}-e^{r_{i}} T\right|^{2} d x
$$

\subsection{Implementation}

We present here briefly the numerical implementation using the manifolds $\mathcal{D}_{i}$. More details can be found in our previous 2-d work [2].

Let $\mathcal{T}$ be the mesh of $D$ used during the optimization process, and $\mathcal{T}_{k}$, its $k^{\text {th }}$ element (a tetrahedron). We recall that we use $P_{1}$ finite elements to discretize all fields, including the vector fields $\omega_{i}$, see figure $12(\mathrm{a})$.

The difficulty is to represent the manifold $\mathcal{D}_{i}$, which is equivalent to two copies of $D$, using finite elements in a classical finite element solver. However, all the maps involved in this problem are either symmetric or antisymmetric on $\mathcal{D}_{i}$. Hence, they are completely determined by their values at $(x, T)$, from which the other values at $(x,-T)$ are deduced by symmetry. As a consequence, a possibility would be to use only one copy of $D$ to represent $\mathcal{D}_{i}$. However, this requires to compute a coherent orientation on the whole domain $D$, a solution we previously declined.

Instead, we subdivide the working domain $D$ into open subsets $U$ whose closures covers $D$. In practice, the tetrahedra $\mathcal{T}_{k}$ of $\mathcal{T}$ are natural candidates for such purpose. Then, we work independently on each tetrahedron and compute a coherent orientation on each tetrahedron, meaning to have a continuous orientation vector field, featuring no change of sign. Hence, on each tetrahedron we can compute the rotational of the orientation vector field. The key point is that we do not require the orientation on each tetrahedron to be coherent with one another. We give more details about coherent orientation in the following.

Recall that standard $P_{1}$ conforming finite elements are used for discretization during the optimization process. However, since we work now on each tetrahedron separately, $P_{1}$ discontinuous Galerkin elements on $\mathcal{T}$ are now used for this post-processing projection. Furthermore symmetry (or antisymmetry) of the considered maps have to be taken into account, which is explained in the following.

Coherent orientation on a tetrahedron. In the whole paragraph, vector fields are discretized by their values at each vertex of the mesh. For a tetrahedron $\mathcal{T}_{k}$ of the mesh $\mathcal{T}$, its four vertices are denoted by $\left(x_{j}\right)_{1 \leq j \leq 4}$. The vector field $\omega_{i}$ on $\mathcal{T}_{k}$ is defined by four vectors. 


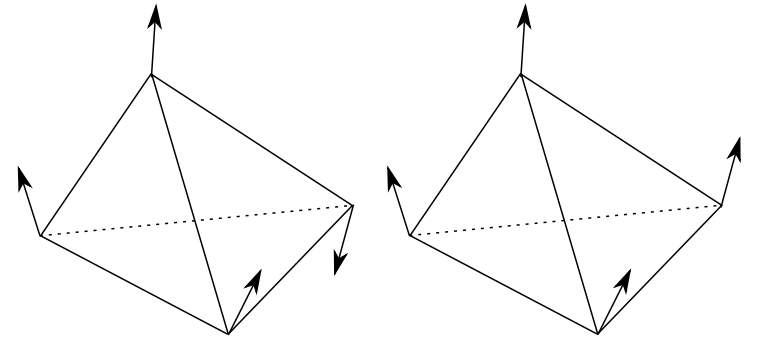

(a) $\omega_{i}$ (b) $\tilde{\omega}_{i}$

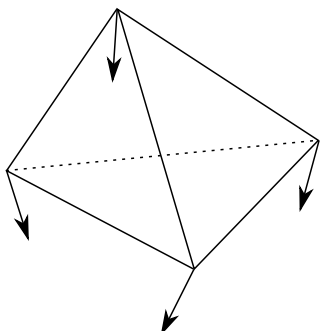

(c) $\tilde{\omega}_{i}$

Figure 11: Optimized orientation field $\omega_{i}$ (a) on a tetrahedron and the both possible coherent orientation fields $\tilde{\omega}_{i}$ from $\omega_{i}$ : the coherent orientation when the first vertex $x_{1}$ is the upper one (b) and the coherent orientation when the first vertex $x_{1}$ is the vertex on the right (c)

A vector field admits a coherent orientation over the tetrahedron $\mathcal{T}_{k}$ if there exists an affine map $\tilde{\omega}_{i}$ from $\mathcal{T}_{k}$ to $\mathbb{R}^{3}$ such that:

$$
\forall j \in\{1,2,3,4\} \quad \text { ' } \tilde{\omega}_{i}\left(x_{j}\right)= \pm \omega_{i}\left(x_{j}\right) \quad \text { and } \quad \forall x \in \mathcal{T}_{k} \quad \tilde{\omega}_{i}(x) \neq 0 .
$$

Such a coherent orientation does not necessarily exists on each tetrahedron. If not, the orientation field is said singular. In the present work, we do not treat such cases and assume that a coherent orientation does indeed exists on every element of the mesh.

In practice, for each tetrahedron, we pick a first vertex $x_{1}$, where the value $\omega_{i}\left(x_{1}\right)$ will be the reference orientation of the tetrahedron : $\tilde{\omega}_{i}\left(x_{1}\right)=\omega_{i}\left(x_{1}\right)$. For the three other vertices $x_{j}$, we choose the orientation $\tilde{\omega}_{i}\left(x_{j}\right)= \pm \omega_{i}\left(x_{j}\right)$ in order to have :

$$
\tilde{\omega}_{i}\left(x_{j}\right) \cdot \tilde{\omega}_{i}\left(x_{1}\right) \geq 0 .
$$

Hence two coherent orientations could be defined on each tetrahedron, see figure (11), depending of the choice of the first vertex $x_{1}$ : one for each copy of $D$ into $\mathcal{D}$.

By iterating this over the whole mesh $\mathcal{T}$ we define a $P_{1}$ discontinuous finite element on $D$. The different steps to construct a coherent orientation $\tilde{\omega}_{i}$ are represented on the Figures 12(a), 12(b) and 12(c). To avoid unnecessary complex figures, that would have undermined their readability, this process is illustrated in a 2 - $d$ setting, which is perfectly similar to the 3 - $d$ case.

Interpolation of symmetric maps. The value of a symmetric map $\psi \in \mathcal{V}_{i}^{s}$ at a vertex does not depend on its second variable, since $\psi(x, T)=\psi(x,-T)$. Hence, it can be represented by a standard conformal $P_{1}$ finite element function over $D$. 
Interpolation of antisymmetric maps. We explain here how to represent an antisymmetric map of $\mathcal{D}_{i}$ using a standard conformal $P_{1}$ finite element function on $D$.

Let $\psi \in \mathcal{V}_{i}^{a}$ be an antisymmetric map. Let $x_{j}$ be a vertex, and $x_{j, k}$ its copies in all tetrahedra featuring $x_{j}$. The value of $\psi$ in each vertex $x_{j, k}$ depends on the current coherent orientation: $\tilde{\omega}_{i}\left(x_{j, k}\right)= \pm \omega_{i}\left(x_{j}\right)$. We can order the vertices $x_{j, k}$ in two sets, $X_{j}^{+}$and $X_{j}^{-}$, according to this sign.

All the elements of $X_{j}^{+}$(resp. of $X_{j}^{-}$) are the same points of the manifold $\mathcal{D}:\left(x_{j}, \omega_{i}\left(x_{j}\right)\right)\left(\operatorname{resp}\left(x_{j},-\omega_{i}\left(x_{j}\right)\right)\right)$. Hence $\psi$ is constant on $X_{j}^{+}$(resp. on $\left.X_{j}^{-}\right)$. Moreover, we have $\psi\left(X_{j}^{+}\right)=-\psi\left(X_{j}^{-}\right)$.

We now have all the requirements to represent the antisymmetric maps by standard $P_{1}$ finite element functions on $D$. Let $I$ be the projection operator from $P_{1}$ elements to $P_{1}$ discontinuous elements. Let $g$ be a $P_{1}$ discontinuous finite element, see figure $12(\mathrm{~d})$ defined by:

$$
g\left(x_{j, k}\right)= \begin{cases}+1 & \text { if } \tilde{\omega}_{i}\left(x_{j, k}\right)=\omega_{i}\left(x_{j}\right) \\ -1 & \text { if } \tilde{\omega}_{i}\left(x_{j, k}\right)=-\omega_{i}\left(x_{j}\right)\end{cases}
$$

Define $I^{a}$, the interpolation operator for antisymmetric maps, from $P_{1}$ finite elements on $\mathcal{D}$ to $P_{1}$ discontinuous finite element on $D$ by $I^{a}=g \times I$, meaning that $I^{a}(\varphi)\left(x_{j, k}\right)=g\left(x_{j, k}\right) \varphi\left(x_{j}\right)$. We emphasize that this operator is uniquely defined, but depends on the previously computed field $\tilde{\omega}_{i}$, the coherent orientation on each tetrahedron.

Computation of $r$ and $\varphi$. In order to compute the dilation factors and the distortion map, as seen previously, we work on each tetrahedron separately, using $P_{1}$ Galerkin discontinuous finite elements. However, as just seen, the three dilation factors $r_{i}$ and the three components of the distortion map $\varphi$ are represented by standard $P_{1}$ finite elements functions, composed with projection operators $I$ and $I^{a}$.

Let $\mathcal{V}$ be the set of $P_{1}$ finite elements on $\mathcal{T}$. The dilation factor $r_{i} \in \mathcal{V}$ is the minimizer of:

$$
\begin{aligned}
\min _{s \in \mathcal{V}} \sum_{\mathcal{T}_{k} \mathcal{T}} \int_{\mathcal{T}_{k}} & \left|\nabla(I(s)) \wedge \tilde{\omega}_{i}+\nabla \wedge \tilde{\omega}_{i}\right|^{2} \\
& +\eta_{1}^{2} \mid \nabla\left(\left.I(s) \cdot \tilde{\omega}_{i}\right|^{2}\right. \\
& +\eta_{2}^{2} \mid\left(\left.I(s)\right|^{2} d x .\right.
\end{aligned}
$$

We emphasize that the $P_{1}$ finite element functions $r_{i}$ is continuous on $\mathcal{T}$.

The $i^{t h}$-component $\varphi_{i} \in \mathcal{V}$ of the distortion map, is the minimizer of:

$$
\min _{\psi \in \mathcal{V}} \sum_{\mathcal{T}_{k} \in \mathcal{T}} \int_{\mathcal{T}_{k}}\left|\nabla\left(I^{a}(\psi)\right)-e^{I\left(r_{i}\right)} \tilde{\omega}_{i}\right|^{2} d x .
$$

Although $I^{a}\left(\varphi_{i}\right)$ is a $P_{1}$ discontinuous finite element function, its absolute value $\left|I^{a}\left(\varphi_{i}\right)\right|=\varphi_{i} \mid$ is continuous on the whole mesh. Since the genuine shapes are 

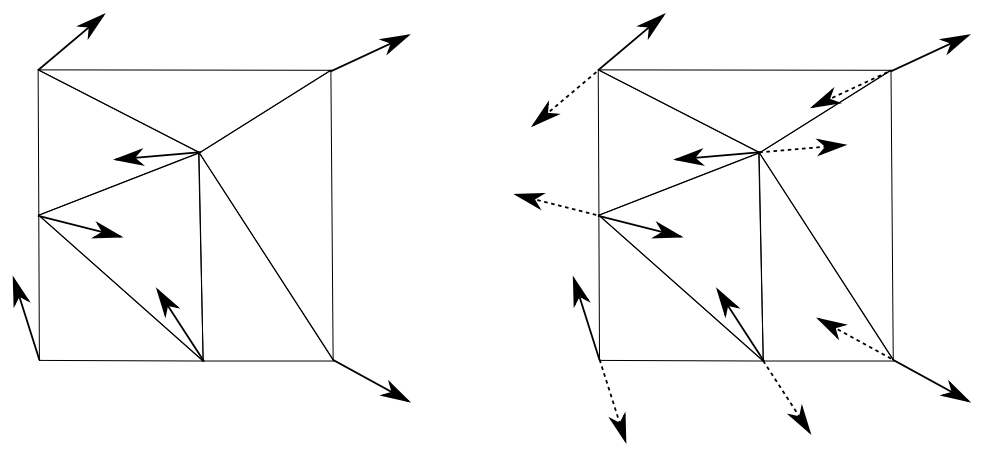

(a) Optimized orientation $\omega_{i}$, defined up to (b) Local possible orientations of the cells its sign $\omega_{i}$ and $-\omega_{i}$, leading to the same final

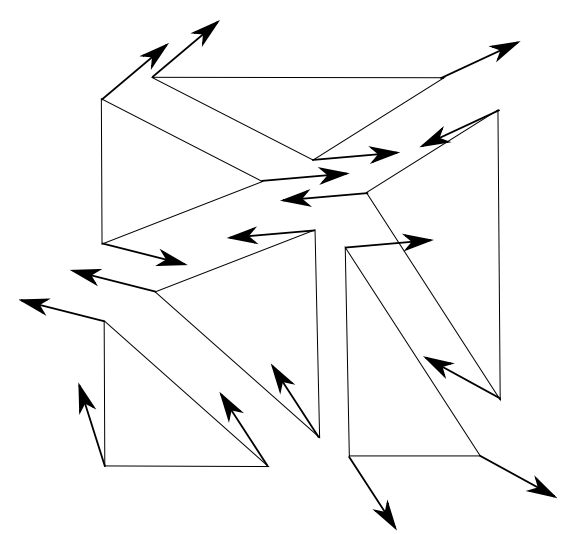
structure

(c) Coherent orientation $\tilde{\omega}_{i}$ as a $P_{1}$ dis- (d) $\tilde{\omega}_{i}$ and the interpolation map $g$ for ancontinuous finite elements tisymmetric maps

Figure 12: Complete process to represent the manifold $\mathcal{D}$ using classical finite elements 
built using the even function cos, the projection is smoothly defined by using $\varphi_{i}$.

Both preceding problems can be classically solved by writing the first order optimality condition, which is a standard variational formulation featuring a linear form and a bilinear form.

\subsection{Numerical results}

The above algorithm for the computation of the diffeomorphism is implemented in Freefem ++ [17]. The projection step has been scripted in Paraview. Hence, stl files of the deshomogenized structures are straightforwardly generated and are ready to be printed using additive manufacturing machines. We emphasizing that the size $\varepsilon$ of the chosen periodic cells is editable on line in order to adapt the resolution of the deshomogenized structures without any supplementary actions.

The cantilever case is chosen in order to assess our method.

The several steps of the whole process of building the distortion of a regular periodic grid are displayed on Figure 13. The regular grid is defined by constant geometric parameters over the whole domain $D: m_{\text {reg }}=(0.9,0.9,0.9)$. Note the definition of the grid is independent of the mesh.

Several shapes $\Omega_{\epsilon}(\varphi, m)$ for various values of the characteristic size $\varepsilon$ of the cells are displayed in Figure 14. The smaller $\varepsilon$, the closer from the homogenized optimal design the shape $\Omega_{\epsilon}(\varphi, m)$.

We emphasize that small non connected components have been removed from the displayed final shapes. The final shapes are therefore connected.

On Figure 14, we displayed several shapes $\Omega_{\epsilon}(\varphi, m)$ for various values of $\epsilon$.

\section{Other numerical examples}

We applied the whole method to a bridge case and to an electrical mast. In both cases, the volume constraint is fixed to $30 \%$ of the working domain and the number of iterations during the optimization - before regularization - is set to 200. The optimized geometric parameters and densities are not displayed for both cases. Indeed, as seen in the cantilever case, such 3-d results are difficult to displayed in 2-d, or required too many figures to be explicit. The main novelties of the present work are the regularization of the orientation and the projection step. Hence we privileged Figures for those parts.

For the bridge (Figure 15) the domain size is $22 \times 10 \times 12$ and a unit vertical load is applied at the middle of the bottom border on a square of length 2 . The Dirichlet boundary condition is applied on two symmetric rectangles, of length 1 and of width 1 , on the bottom border. The resulting compliance for the homogenized design is 1.017 .

The applied loads and the boundary conditions are displayed on Figure 15, the optimal orientation of the periodicity cells before and after regularization on Figure 16. The distortion of a regular grid is displayed on Figure 17. The sequence of final shapes is displayed on Figure 18. 


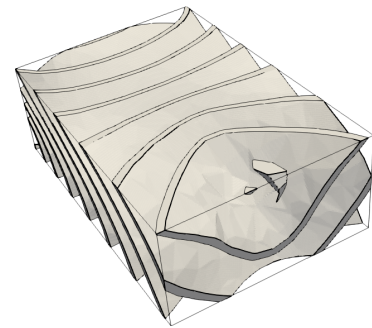

(a) $\Omega_{1}=\Omega_{1, \varphi, 0.2}\left(m_{r e g}\right)$

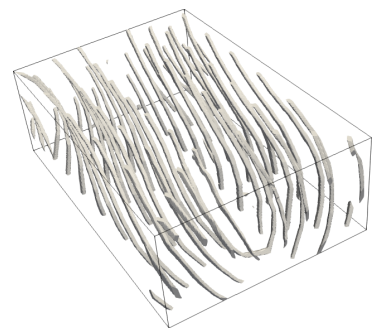

(d) $\Omega_{12}=\Omega_{1} \cap \Omega_{2}$

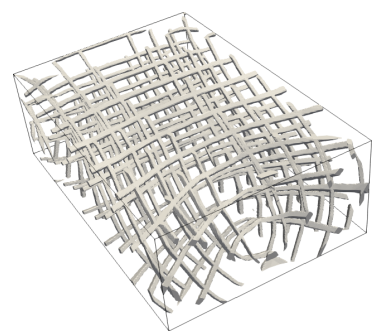

(g) $\Omega_{12} \cup \Omega_{13}$

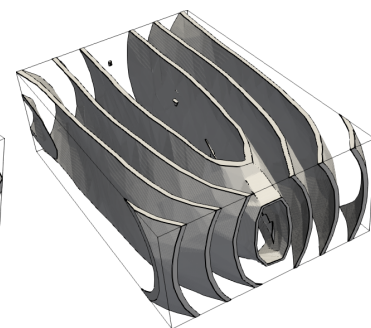

(b) $\Omega_{2}=\Omega_{2, \varphi, 0.2}\left(m_{r e g}\right)$

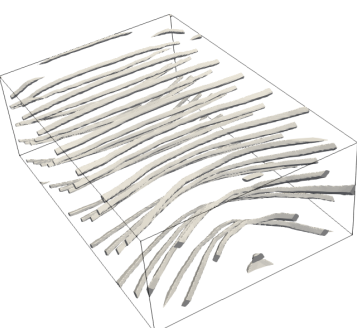

(e) $\Omega_{13}=\Omega_{1} \cap \Omega_{3}$

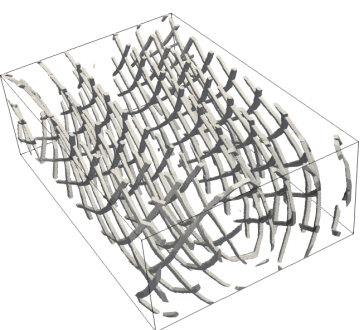

(h) $\Omega_{12} \cup \Omega_{23}$

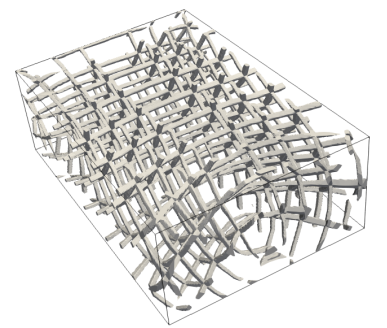

(j) $\Omega_{12} \cup \Omega_{13} \cup \Omega_{23}$

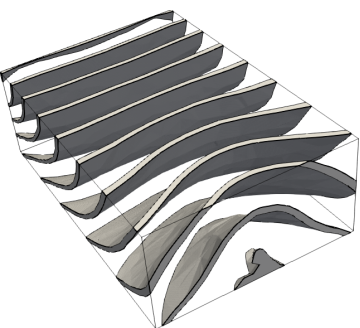

(c) $\Omega_{3}=\Omega_{3, \varphi, 0.2}\left(m_{r e g}\right)$

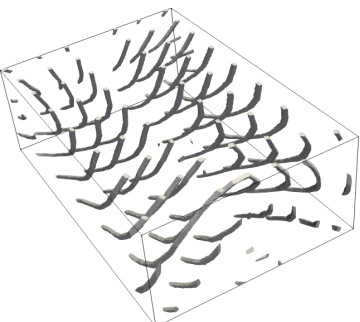

(f) $\Omega_{23}=\Omega_{2} \cap \Omega_{3}$

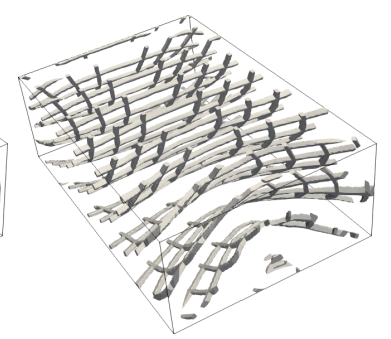

(i) $\Omega_{13} \cup \Omega_{23}$

Figure 13: Distortion of a regular grid through the map $\varphi$ from the cantilever case 


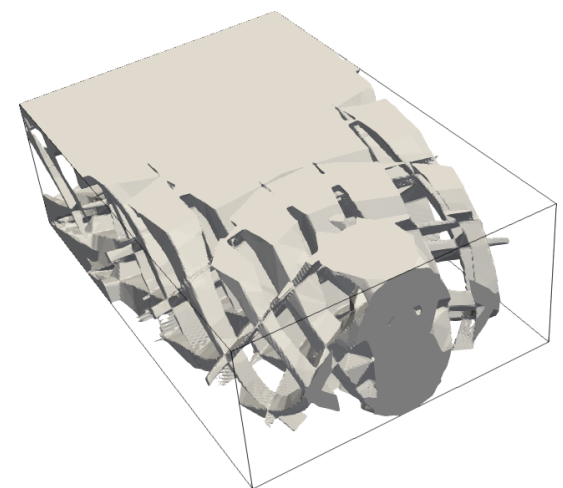

(a) $\epsilon=0.275$

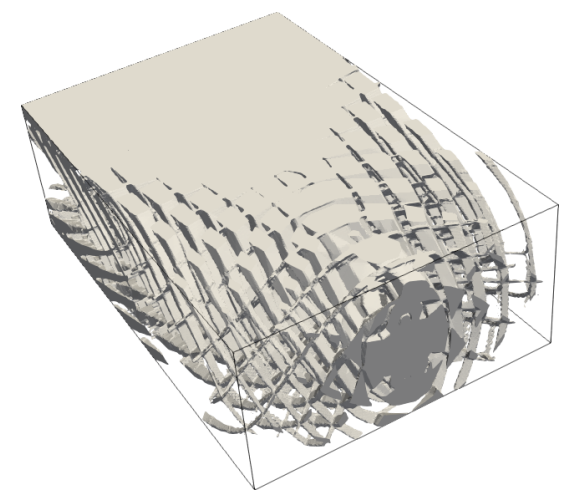

(c) $\epsilon=0.125$

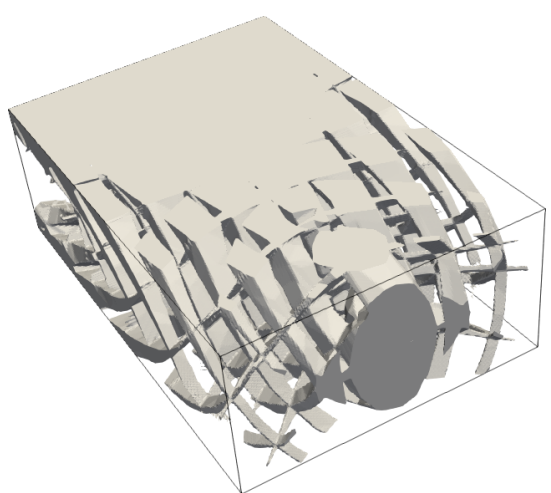

(b) $\epsilon=0.225$

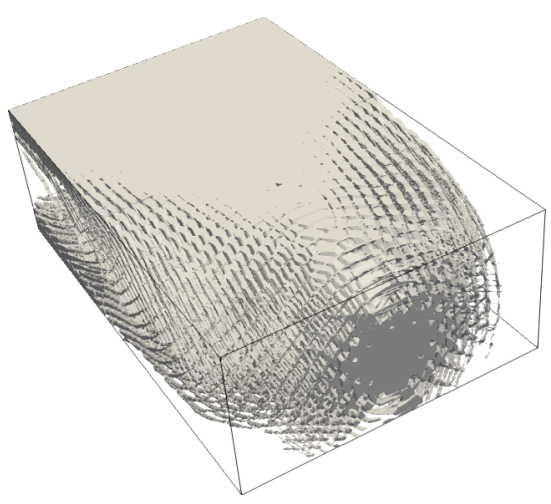

(d) $\epsilon=0.05$

Figure 14: $\Omega_{\epsilon}(\varphi, m)$ for several $\epsilon$ in the case of the cantilever 


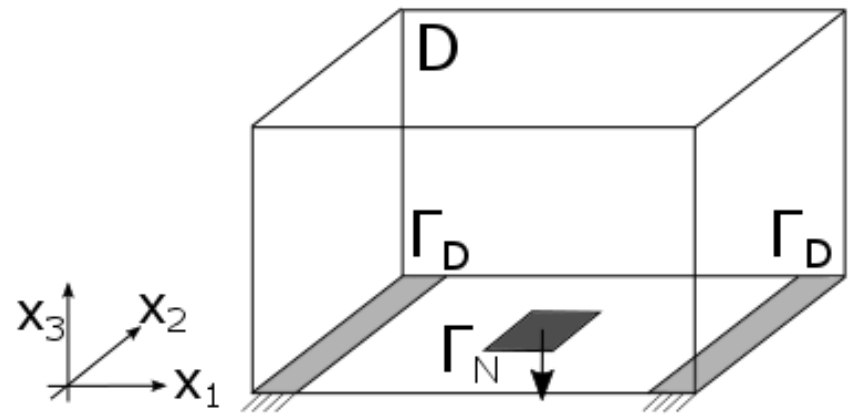

Figure 15: Boundary conditions for the bridge case

For the electrical mast (Figure 19), the domain size of the pillar is $2 \times 2 \times 8$, and the domain size of the upper part is $9 \times 9 \times 3.5$. We took advantage of the symmetry, by running the algorithm just on the quarter of a complete working domain : the domain in bold on Figure 19. Then the structure is allowed to slide on its two intern sides. The Dirichlet boundary condition is applied on the bottom border. A unit vertical load is applied on a square of size 1 on the external corner of the bottom face of the upper part of the domain. The resulting compliance for the homogenized design on the quarter of the whole domain is 4.288 .

The optimal orientation of the periodicity cells before and after regularization is displayed on Figure 20. The distortion of a regular grid is displayed on Figure 20. The sequence of final shapes is displayed on Figure 21, for the quarter of the domain and for the whole domain : the second ones are obtained by reflections of the first ones. The pillar is not completely full, but feature thin holes: central slices of final structures and of normal $x_{1}$ are displayed on Figure 22. We emphasize that nothing ensures that the phase of $\varphi$ is the same for each value of $\varepsilon$ : on a given slice, the density could seem to be not the same according to the value of $\varepsilon$, but it is not the case.

\section{Appendix}

In numerical practice, we use the normalized Voigt notations. Hence a symmetric tensor $s$ of order 2 , like the stress or strain tensor, is represented by a vector $\{s\}$ of size 6 with same norm:

$$
\{s\}=\left(\begin{array}{c}
s_{11} \\
s_{22} \\
s_{33} \\
\sqrt{2} s_{12} \\
\sqrt{2} s_{13} \\
\sqrt{2} s_{23}
\end{array}\right) \text {. }
$$




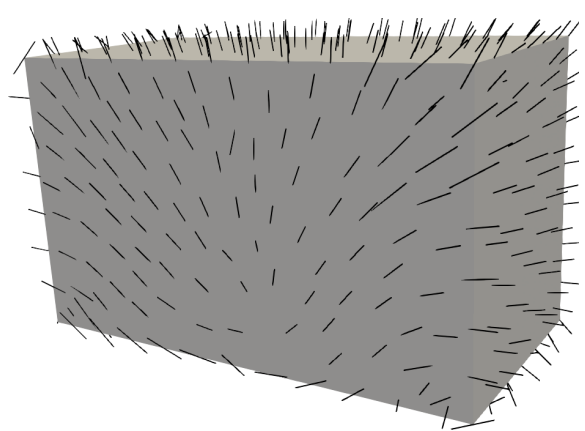

(a) Optimized $\omega_{1}$

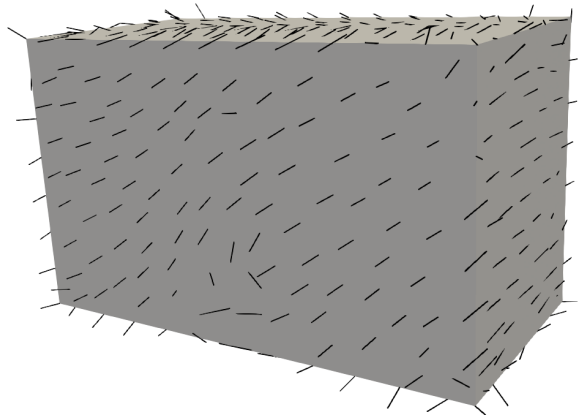

(c) Optimized $\omega_{2}$

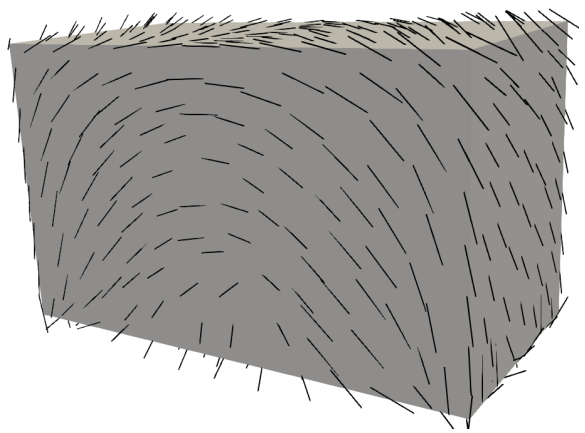

(e) Optimized $\omega_{3}$

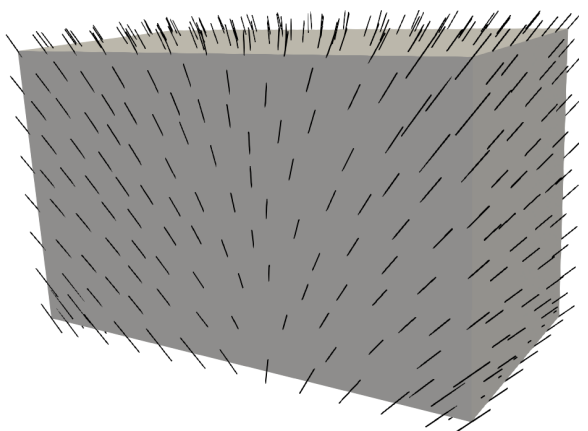

(b) Regularized $\omega_{1}$

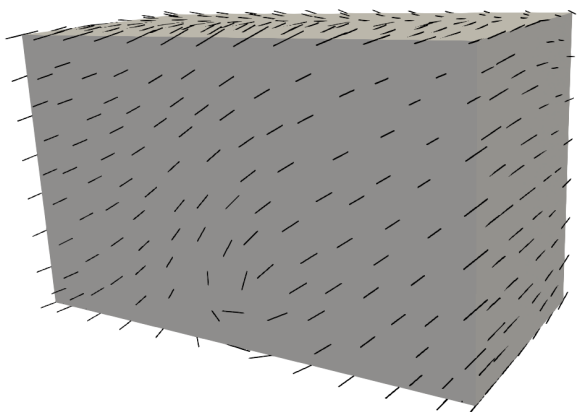

(d) Regularized $\omega_{2}$

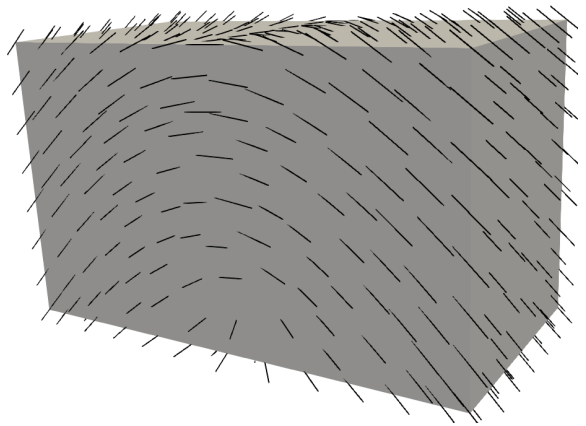

(f) Regularized $\omega_{3}$

Figure 16: Optimized (left) and regularized (right) orientation for the bridge case 


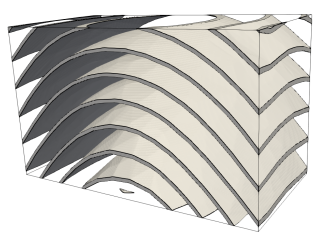

(a) $\Omega_{1}=\Omega_{1, \varphi, 0.2}\left(m_{r e g}\right)$

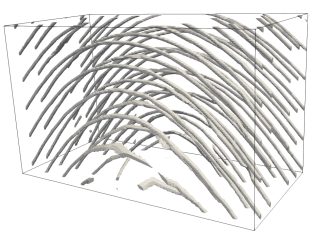

(d) $\Omega_{12}=\Omega_{1} \cap \Omega_{2}$

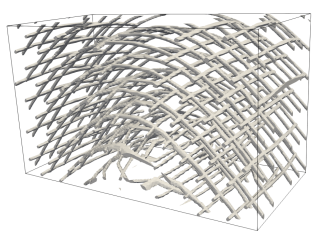

(g) $\Omega_{12} \cup \Omega_{13}$

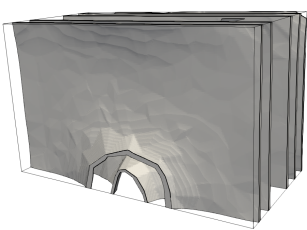

(b) $\Omega_{2}=\Omega_{2, \varphi, 0.2}\left(m_{r e g}\right)$

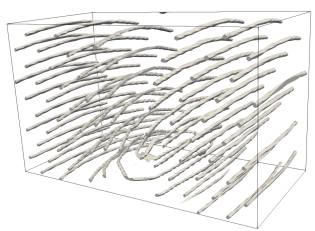

(e) $\Omega_{13}=\Omega_{1} \cap \Omega_{3}$

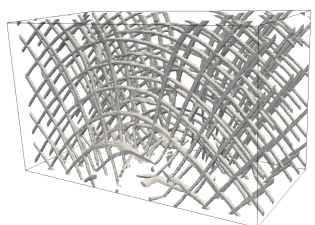

(h) $\Omega_{12} \cup \Omega_{23}$

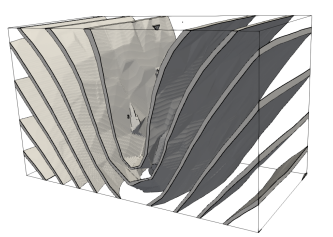

(c) $\Omega_{3}=\Omega_{3, \varphi, 0.2}\left(m_{r e g}\right)$

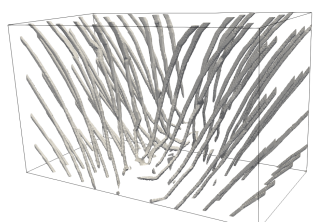

(f) $\Omega_{23}=\Omega_{2} \cap \Omega_{3}$

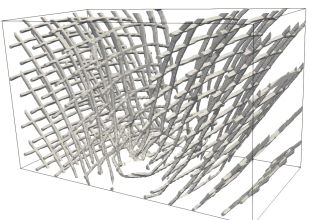

(i) $\Omega_{13} \cup \Omega_{23}$

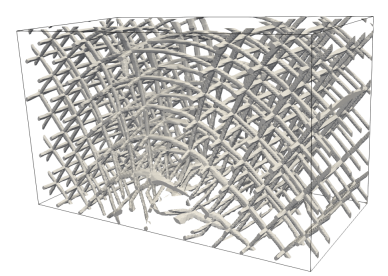

(j) $\Omega_{12} \cup \Omega_{13} \cup \Omega_{23}$

Figure 17: Distortion of a regular grid through the map $\varphi$ from the bridge case 


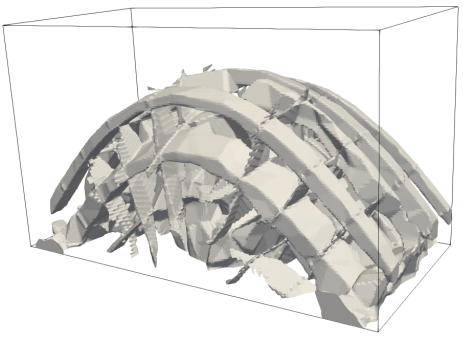

(a) $\epsilon=0.5$

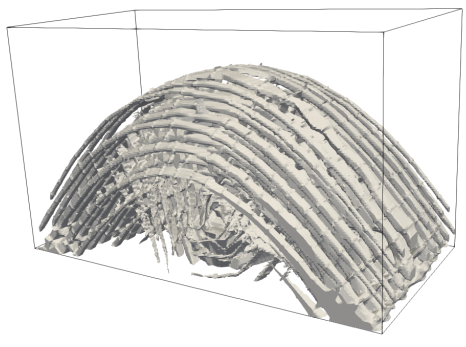

(c) $\epsilon=0.2$

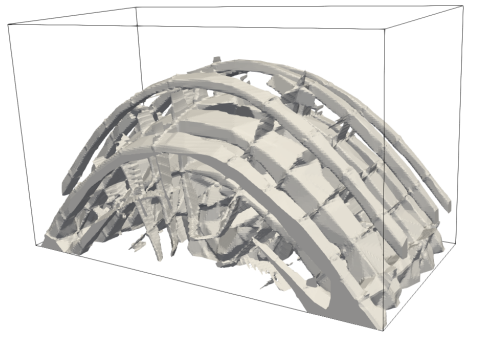

(b) $\epsilon=0.4$

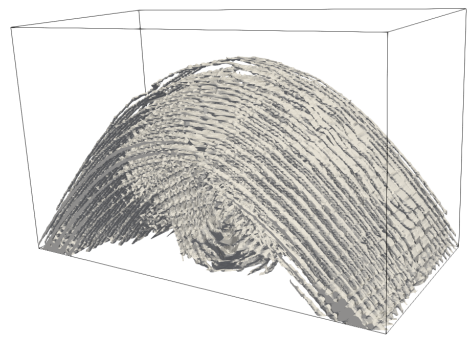

(d) $\epsilon=0.1$

Figure 18: $\Omega_{\epsilon}(\varphi, m)$ for several $\epsilon$ in the case of the bridge

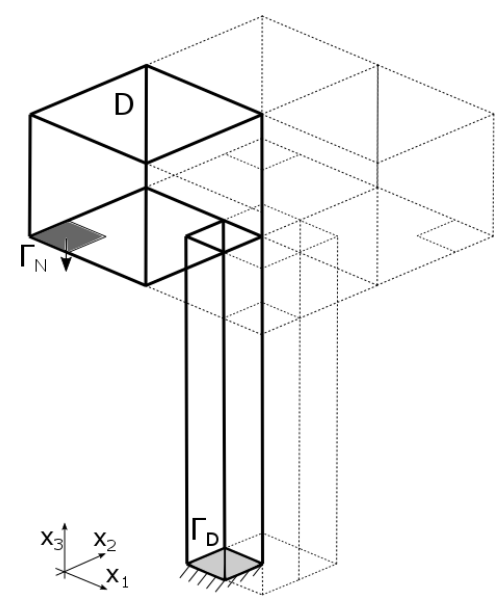

Figure 19: Boundary conditions for the bridge case 


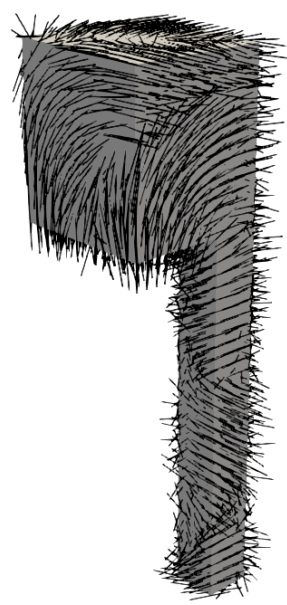

(a) Optimized $\omega_{1}$

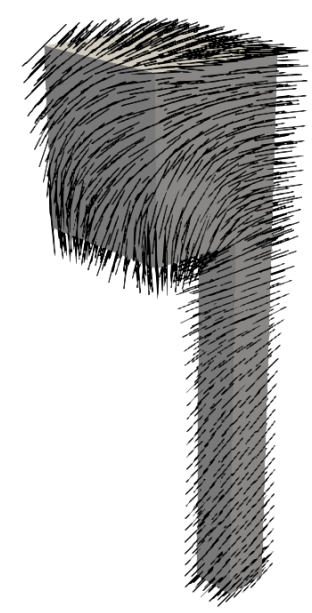

(d) Regularized $\omega_{1}$

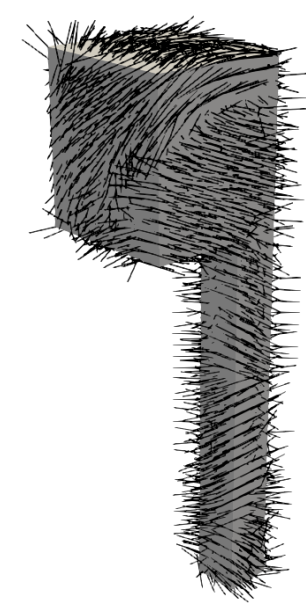

(b) Optimized $\omega_{2}$

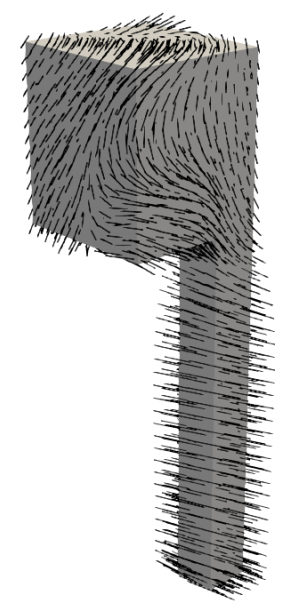

(e) Regularized $\omega_{2}$

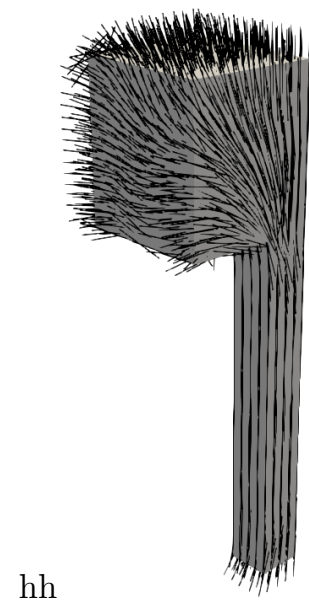

(c) Optimized $\omega_{3}$

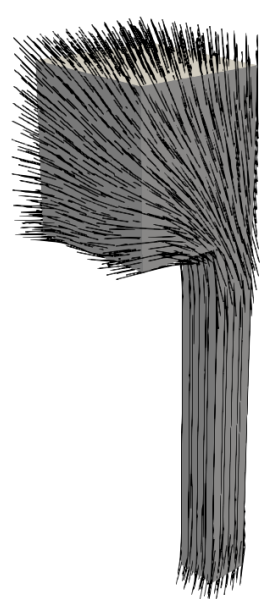

(f) Regularized $\omega_{3}$

Figure 20: Optimized (up) and regularized (down) orientation for the electrical mast case 


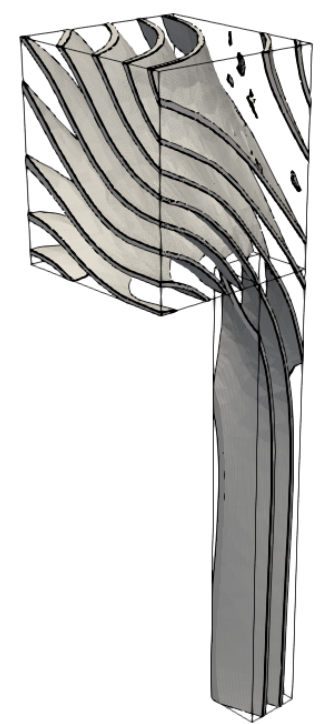

(a) $\Omega_{1}=\Omega_{1, \varphi, \varepsilon}\left(m_{\mathrm{reg}}\right)$

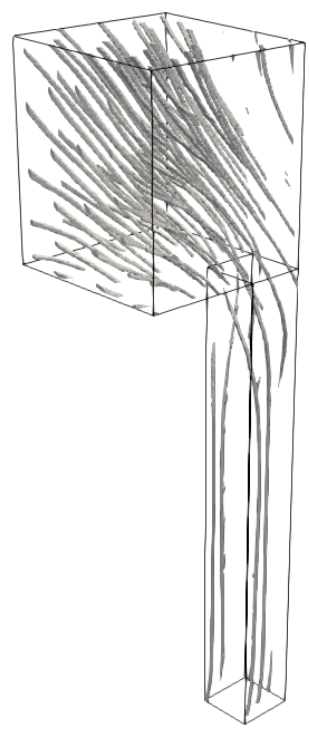

(d) $\Omega_{12}=\Omega_{1} \cap \Omega_{2}$

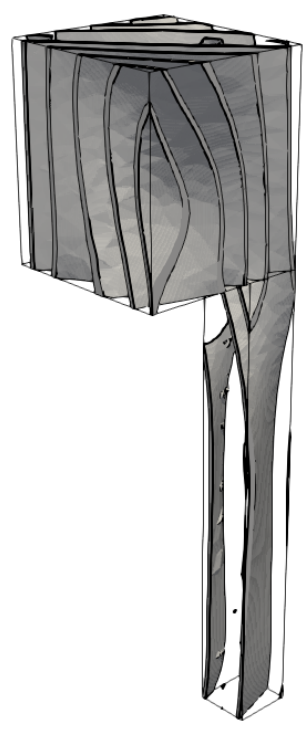

(b) $\Omega_{2}=\Omega_{2, \varphi, \varepsilon}\left(m_{\mathrm{reg}}\right)$

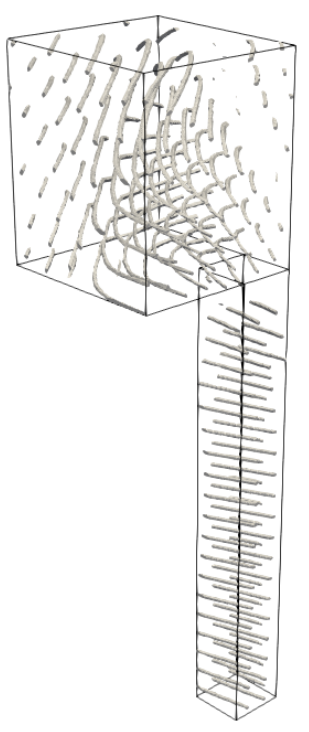

(e) $\Omega_{13}=\Omega_{1} \cap \Omega_{3}$

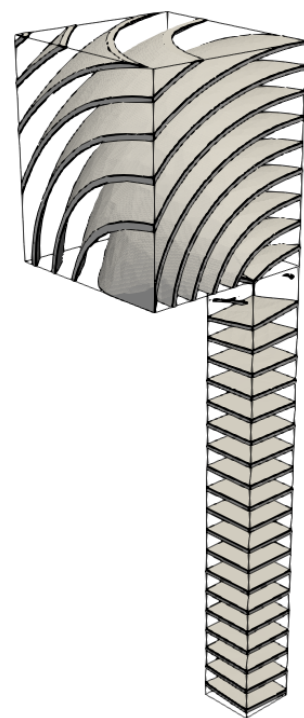

(c) $\Omega_{3}=\Omega_{3, \varphi, \varepsilon}\left(m_{\mathrm{reg}}\right)$

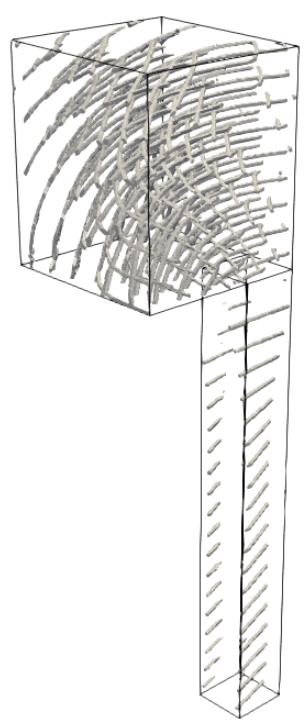

(f) $\Omega_{23}=\Omega_{2} \cap \Omega_{3}$ 


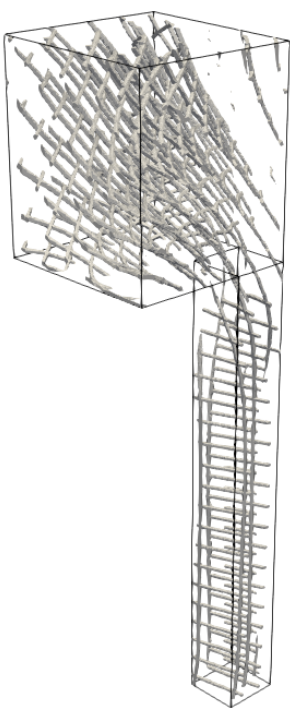

(g) $\Omega_{12} \cup \Omega_{13}$

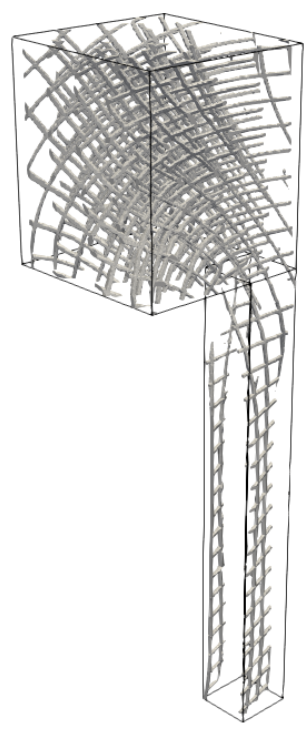

(h) $\Omega_{12} \cup \Omega_{23}$

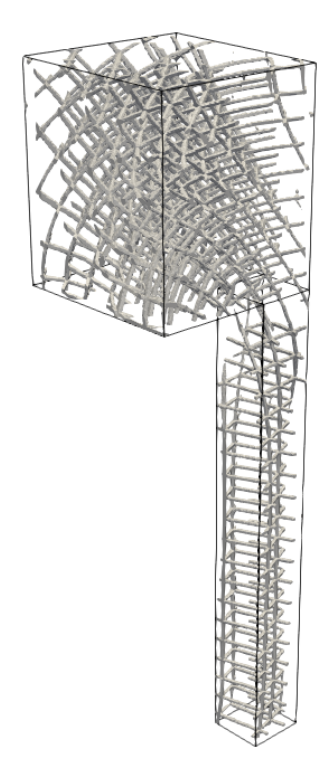

(j) $\Omega_{12} \cup \Omega_{13} \cup \Omega_{23}$

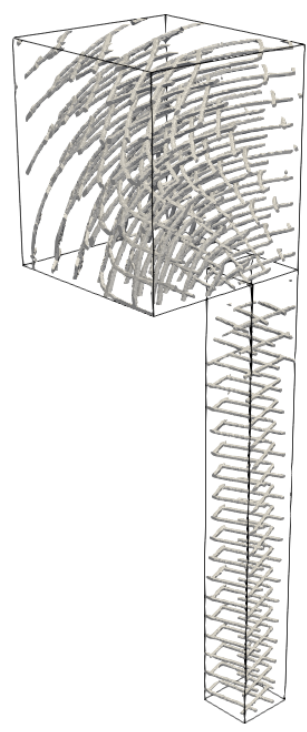

(i) $\Omega_{13} \cup \Omega_{23}$

Figure 20: Distortion of a regular grid through the map $\varphi$ from the electrical mast test case 


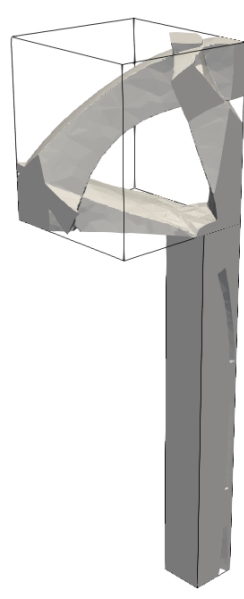

(k) $\epsilon=1$.

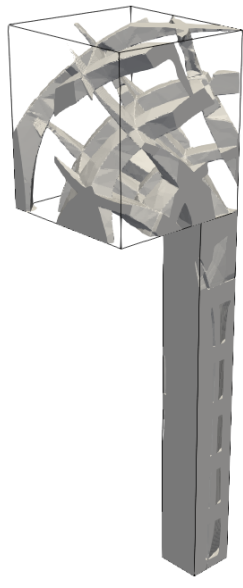

(l) $\epsilon=0.4$

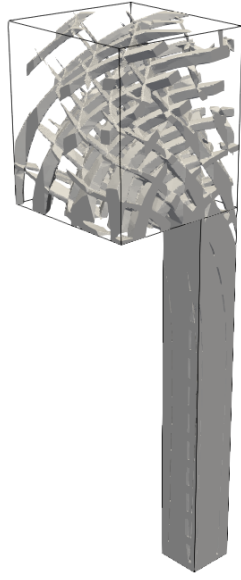

(m) $\epsilon=0.2$

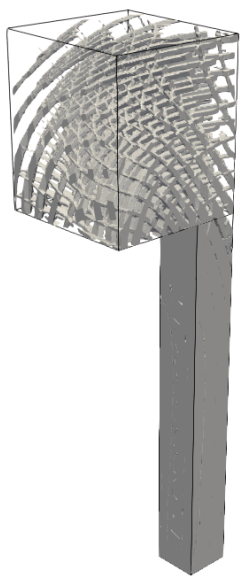

(n) $\epsilon=0.1$

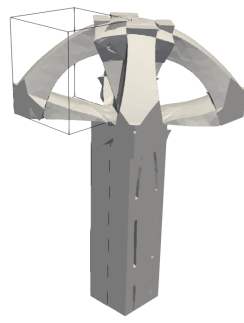

(o) $\epsilon=1$.

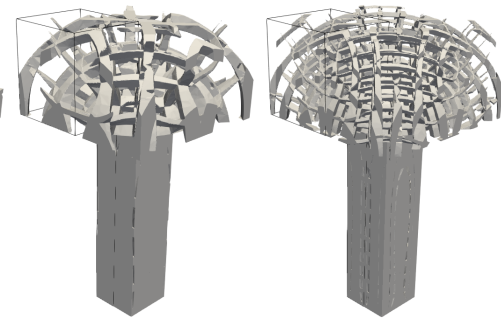

(p) $\epsilon=0.4$

(q) $\epsilon=0.2$

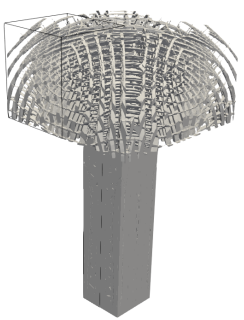

(r) $\epsilon=0.1$

Figure 21: $\Omega_{\epsilon}(\varphi, m)$ for several $\epsilon$ in the electrical mast test case on the quarter of the whole domain (up), and on the whole domain (down).

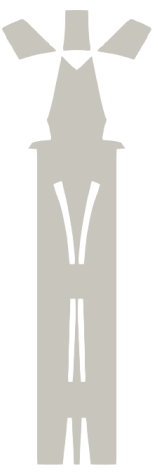

(a) $\epsilon=1$.

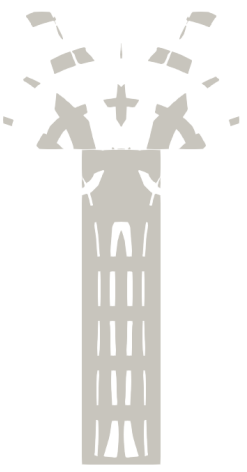

(b) $\epsilon=0.4$

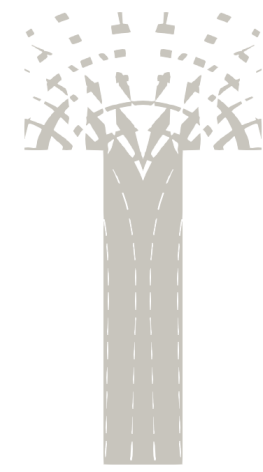

(c) $\epsilon=0.2$

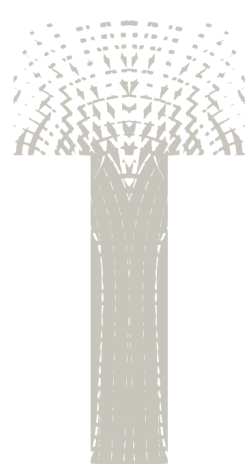

(d) $\epsilon=0.1$

Figure 22: Slices of normal $x_{1}$ of $\Omega_{\epsilon}(\varphi, m)$ for several $\epsilon$ for the electrical mast test. 
The rotation operator $R(\omega)$, as any fourth-order tensor, is then represented by a $6 \times 6$ matrix, which is precisely defined by

$$
\{R(\omega)\}=\left(\begin{array}{ll}
R_{11}(\omega) & R_{12}(\omega) \\
R_{21}(\omega) & R_{22}(\omega)
\end{array}\right),
$$

where the four sub-matrices $R_{i j}$ of size $3 \times 3$ are respectively given by:

$$
\begin{gathered}
R_{11}(\omega)=\left(\begin{array}{lll}
\omega_{1,1}^{2} & \omega_{2,1}^{2} & \omega_{3,1}^{2} \\
\omega_{1,2}^{2} & \omega_{2,2}^{2} & \omega_{3,2}^{2} \\
\omega_{1,3}^{2} & \omega_{2,3}^{2} & \omega_{3,3}^{2}
\end{array}\right) \\
R_{12}(\omega)=\left(\begin{array}{lll}
\sqrt{2} \omega_{1,1} \omega_{2,1} & \sqrt{2} \omega_{1,1} \omega_{3,1} & \sqrt{2} \omega_{2,1} \omega_{3,1} \\
\sqrt{2} \omega_{1,2} \omega_{2,2} & \sqrt{2} \omega_{1,2} \omega_{3,2} & \sqrt{2} \omega_{2,2} \omega_{3,2} \\
\sqrt{2} \omega_{1,3} \omega_{2,3} & \sqrt{2} \omega_{1,3} \omega_{3,3} & \sqrt{2} \omega_{2,3} \omega_{3,3}
\end{array}\right), \\
R_{21}(\omega)=\left(\begin{array}{lll}
\sqrt{2} \omega_{1,1} \omega_{1,2} & \sqrt{2} \omega_{2,1} \omega_{2,2} & \sqrt{2} \omega_{3,1} \omega_{3,2} \\
\sqrt{2} \omega_{1,1} \omega_{1,3} & \sqrt{2} \omega_{2,1} \omega_{2,3} & \sqrt{2} \omega_{3,1} \omega_{3,3} \\
\sqrt{2} \omega_{1,2} \omega_{1,3} & \sqrt{2} \omega_{2,2} \omega_{2,3} & \sqrt{2} \omega_{3,2} \omega_{3,3}
\end{array}\right), \\
R_{22}(\omega)=\left(\begin{array}{lll}
\omega_{1,1} \omega_{2,2}+\omega_{1,2} \omega_{2,1} & \omega_{1,1} \omega_{3,2}+\omega_{1,2} \omega_{3,1} & \omega_{2,1} \omega_{3,2}+\omega_{2,2} \omega_{3,1} \\
\omega_{1,1} \omega_{2,3}+\omega_{1,3} \omega_{2,1} & \omega_{1,1} \omega_{3,3}+\omega_{1,3} \omega_{3,1} & \omega_{2,1} \omega_{3,3}+\omega_{2,3} \omega_{3,1} \\
\omega_{1,3} \omega_{2,2}+\omega_{1,2} \omega_{2,3} & \omega_{1,3} \omega_{3,2}+\omega_{1,2} \omega_{3,3} & \omega_{2,3} \omega_{3,2}+\omega_{2,2} \omega_{3,3}
\end{array}\right) .
\end{gathered}
$$

\section{Acknowledgments}

P. G. D. is a CIFRE PhD student, funded by ANRT ( $\left.{ }^{\circ} 2015 / 0770\right)$, the support of which is kindly acknowledged. G. A. is a member of the DEFI project at INRIA Saclay Ile-de-France. The work of G. A. is partially supported by the SOFIA project, funded by BPI (Banque Publique d'Investissement).

\section{References}

[1] G. Allaire. Shape optimization by the homogenization method, volume 146. Springer Science \& Business Media, 2002.

[2] G. Allaire, P. Geoffroy-Donders, and O. Pantz. Topology optimization of modulated and oriented periodic microstructures by the homogenization method. Computers and Mathematics with Applications, 2018.

[3] G. Allaire and O. Pantz. Structural optimization with freefem++. Structural and Multidisciplinary Optimization, 32(3):173-181, 2006.

[4] S. Aubry. Etude théorique et numérique de quelques problèmes d'optimisation de forme à l'aide de méthodes d'homogénéisation. $\mathrm{PhD}$ thesis, Université Pierre et Marie Curie, 1999. 
[5] C. Barbarosie. Optimization of perforated domains through homogenization. Structural optimization, 14(4):225-231, 1997.

[6] C. Barbarosie and A.-M. Toader. Shape and topology optimization for periodic problems. Structural and Multidisciplinary Optimization, 40(1):393408, 2010.

[7] C. Barbarosie and A.-M. Toader. Shape and topology optimization for periodic problems. II. Optimization algorithm and numerical examples. Struct. Multidiscip. Optim., 40(1-6):393-408, 2010.

[8] M. P. Bendsøe. Optimal shape design as a material distribution problem. Structural and multidisciplinary optimization, 1(4):193-202, 1989.

[9] M. P. Bendsøe and N. Kikuchi. Generating optimal topologies in structural design using a homogenization method. Computer methods in applied mechanics and engineering, 71(2):197-224, 1988.

[10] M. P. Bendsoe and O. Sigmund. Topology optimization: theory, methods, and applications. Springer Science \& Business Media, 2003.

[11] A. D. Cramer, V. J. Challis, and A. P. Roberts. Microstructure interpolation for macroscopic design. Structural and Multidisciplinary Optimization, 53(3):489-500, 2016.

[12] A. D. Cramer, V. J. Challis, and A. P. Roberts. Physically realisable 3d bone prosthesis design with interpolated microstructures. Journal of Biomechanical Engineering, 2016.

[13] V. Dolean, P. Jolivet, and F. Nataf. An introduction to domain decomposition methods: algorithms, theory, and parallel implementation, volume 144. SIAM, 2015.

[14] P. Geoffroy. Homogenization method for topology optimization of structures built with lattice materials. $\mathrm{PhD}$ thesis, École Polytechique, Palaiseau, France, 2018.

[15] J. P. Groen and O. Sigmund. Homogenization-based topology optimization for high-resolution manufacturable micro-structures. International Journal for Numerical Methods in Engineering, 2017.

[16] J. Haslinger and J. Dvořák. Optimum composite material design. ESAIM: Mathematical Modelling and Numerical Analysis, 29(6):657-686, 1995.

[17] F. Hecht. New development in freefem++. Journal of numerical mathematics, 20(3-4):251-266, 2012.

[18] S. A. Khanoki and D. Pasini. Multiscale design and multiobjective optimization of orthopedic hip implants with functionally graded cellular material. Journal of biomechanical engineering, 134(3):031004, 2012. 
[19] R. V. Kohn and G. Strang. Optimal design and relaxation of variational problems. I. Comm. Pure Appl. Math., 39(1):113-137, 1986.

[20] K. Li, X.-L. Gao, and G. Subhash. Effects of cell shape and strut crosssectional area variations on the elastic properties of three-dimensional opencell foams. Journal of the Mechanics and Physics of Solids, 54(4):783-806, 2006.

[21] R. Lipton. Design of functionally graded composite structures in the presence of stress constraints. International journal of solids and structures, $39(9): 2575-2586,2002$.

[22] L. Luo, Y. Fan, and D. Tondeur. Heat exchanger: from micro-to multi-scale design optimization. International journal of energy research, 31(13):1266$1274,2007$.

[23] K. A. Lurie, A. V. Cherkaev, and A. V. Fedorov. Regularization of optimal design problems for bars and plates. I, II. J. Optim. Theory Appl., 37(4):499-522, 523-543, 1982.

[24] J. Martínez, H. Song, J. Dumas, and S. Lefebvre. Orthotropic k-nearest foams for additive manufacturing. ACM Transactions on Graphics (TOG), 36(4):121, 2017.

[25] F. Murat and L. Tartar. Calcul des variations et homogénéisation. In Homogenization methods: theory and applications in physics (Bréau-sansNappe, 1983), volume 57 of Collect. Dir. Études Rech. Élec. France, pages 319-369. Eyrolles, Paris, 1985.

[26] A. N. Norris. Optimal orientation of anisotropic solids. The Quarterly Journal of Mechanics and Applied Mathematics, 59(1):29-53, 2005.

[27] O. Pantz and K. Trabelsi. A post-treatment of the homogenization method for shape optimization. SIAM Journal on Control and Optimization, 47(3):1380-1398, 2008.

[28] P. Pedersen. On optimal orientation of orthotropic materials. Structural Optimization, 1(2):101-106, 1989.

[29] J. G. Rešetnjak. Liouville's conformal mapping theorem under minimal regularity hypotheses. Sibirsk. Mat. Ž., 8:835-840, 1967.

[30] H. Rodrigues, J. Guedes, and M. Bendsoe. Hierarchical optimization of material and structure. Struct Multidisc Optim, 24:1-10, 2002.

[31] C. Schumacher, B. Bickel, J. Rys, S. Marschner, C. Daraio, and M. Gross. Microstructures to control elasticity in $3 \mathrm{~d}$ printing. ACM Transactions on Graphics (TOG), 34(4):136, 2015. 
[32] W. Wang, T. Y. Wang, Z. Yang, L. Liu, X. Tong, W. Tong, J. Deng, F. Chen, and X. Liu. Cost-effective printing of 3d objects with skin-frame structures. ACM Transactions on Graphics (TOG), 32(6):177, 2013.

[33] Y. Wang, H. Xu, and D. Pasini. Multiscale isogeometric topology optimization for lattice materials. Comput. Methods Appl. Mech. Engrg., 316:568-585, 2017.

[34] Q. Xia and M. Y. Wang. Simultaneous optimization of the material properties and the topology of functionally graded structures. Computer-Aided Design, 40(6):660-675, 2008.

[35] M. Zhou and G. Rozvany. The coc algorithm, part ii: Topological, geometrical and generalized shape optimization. Computer Methods in Applied Mechanics and Engineering, 89(1-3):309 - 336, 1991. Second World Congress on Computational Mechanics.

[36] S. Zhou and Q. Li. Design of graded two-phase microstructures for tailored elasticity gradients. Journal of Materials Science, 43(15):5157, 2008. 